\title{
LITTLEWOOD-PALEY FORMULAS AND CARLESON MEASURES FOR WEIGHTED FOCK SPACES INDUCED BY $A_{\infty}$-TYPE WEIGHTS
}

\author{
CARME CASCANTE, JOAN FÀBREGA, AND JOSÉ A. PELÁEZ
}

\begin{abstract}
We obtain Littlewood-Paley formulas for Fock spaces $\mathcal{F}_{\beta, \omega}^{q}$ induced by weights $\omega \in A_{\infty}^{\text {restricted }}=\cup_{1 \leq p<\infty} A_{p}^{\text {restricted }}$, where $A_{p}^{\text {restricted }}$ is the class of weights such that the Bergman projection $P_{\alpha}$, on the classical Fock space $\mathcal{F}_{\alpha}^{2}$, is bounded on

$$
\mathcal{L}_{\alpha, \omega}^{p}:=\left\{f: \int_{\mathbb{C}}|f(z)|^{p} e^{-p \frac{\alpha}{2}|z|^{2}} \omega(z) d A(z)<\infty\right\} .
$$

Using these equivalent norms for $\mathcal{F}_{\beta, \omega}^{q}$ we characterize the Carleson measures for weighted Fock-Sobolev spaces $\mathcal{F}_{\beta, \omega}^{q, n}$.
\end{abstract}

\section{INTRODUCTION}

Let $\mathbb{C}$ be the complex plane and denote by $H(\mathbb{C})$ the space of entire functions. For $0<p, \alpha<\infty$, let $\mathcal{L}_{\alpha}^{p}$ be the space of measurable functions such that

$$
\|f\|_{\mathcal{L}_{\alpha}^{p}}^{p}:=\frac{p \alpha}{2 \pi} \int_{\mathbb{C}}|f(z)|^{p} e^{-p \frac{\alpha}{2}|z|^{2}} d A(z)<\infty,
$$

where $d A$ denotes the Lebesgue measure in $\mathbb{C}$. The classical Fock spaces $\mathcal{F}_{\alpha}^{p}$ consists of the $f \in H(\mathbb{C})$ such that $f \in \mathcal{L}_{\alpha}^{p}[19,21]$. A function $\omega: \mathbb{C} \rightarrow$ $[0, \infty)$ is a weight if $\omega$ belongs to $L_{l o c}^{1}(\mathbb{C}, d A)$. In this paper, we will deal with the weighted spaces of measurable functions,

$$
\mathcal{L}_{\alpha, \omega}^{p}:=\left\{f:\|f\|_{\mathcal{L}_{\alpha, \omega}^{p}}^{p}=\int_{\mathbb{C}}|f(z)|^{p} e^{-p \frac{\alpha}{2}|z|^{2}} \omega(z) d A(z)<\infty\right\},
$$

and the weighted Fock spaces $\mathcal{F}_{\alpha, \omega}^{p}=\mathcal{L}_{\alpha, \omega}^{p} \cap H(\mathbb{C})$.

Date: July 24, 2018.

2010 Mathematics Subject Classification. 30H20, 42B25, 46E35.

Key words and phrases. Fock spaces, Littlewood-Paley formula, Carleson measures, pointwise multipliers.

The research of the first two authors was supported in part by Ministerio de Economía y Competitividad, Spain, projects MTM2014-51834-P and MTM2015-69323-REDT, and Generalitat de Catalunya, project 2014SGR289.

The research of the third author was supported in part by Ministerio de Economía y Competitividad, Spain, projects MTM2014-52865-P, and MTM2015-69323-REDT; La Junta de Andalucía, project FQM210. 
The orthogonal projection $P_{\alpha}: \mathcal{L}_{\alpha}^{2} \rightarrow \mathcal{F}_{\alpha}^{2}$ coincides with the integral operator

$$
P_{\alpha}(f)(z):=\frac{\alpha}{\pi} \int_{\mathbb{C}} f(\zeta) e^{\alpha \bar{\zeta} z} e^{-\alpha|\zeta|^{2}} d A(\zeta), \quad f \in \mathcal{L}_{\alpha}^{2} .
$$

The boundedness of projections on $L^{p}$-spaces is a classical and interesting topic, with a flurry of activity in the recent years, which has plenty of applications on operator theory $[1,2,10,17,20,21]$. It is known that $P_{\alpha}$, and the positive linear operator

$$
P_{\alpha}^{+}(f)(z):=\frac{\alpha}{\pi} \int_{\mathbb{C}} f(\zeta)\left|e^{\alpha \bar{\zeta} z}\right| e^{-\alpha|\zeta|^{2}} d A(\zeta), \quad f \in \mathcal{L}_{\alpha}^{2},
$$

are bounded on the classical $L^{p}\left(\mathbb{C}, e^{-\alpha|\cdot|^{2}} d A\right)$ if and only if $p=2$. However they are bounded on $\mathcal{L}_{\alpha}^{p}$ for any $p \geq 1$, [21, Theorem 2.20] (see also [19]). Recently, the weights such that $P_{\alpha}$ is bounded on $\mathcal{L}_{\alpha, \omega}^{p}$ have been described in [10, Theorem 3.1]. In order to state this result, we need a bit more of notation. If $E \subset \mathbb{C}$ is measurable, we denote by $|E|$ its Lebesgue area measure and let $\omega(E):=\int_{E} \omega(z) d A(z)$. Throughout the paper $Q$ denotes a square in $\mathbb{R}^{2}$. We write $l(Q)$ for its side length and as usual, $\frac{1}{p}+\frac{1}{p^{\prime}}=1$, for $1 \leq p \leq \infty$.

Let $A_{p, r}$ be the class of weights $\omega$ on $\mathbb{C}$ such that $\omega(z)>0$ a.e. on $\mathbb{C}$ and

$$
\mathcal{C}_{p, r}(\omega):=\sup _{Q, l(Q)=r}\left(\frac{1}{|Q|} \int_{Q} \omega d A\right)\left(\frac{1}{|Q|} \int_{Q} \omega^{-\frac{p^{\prime}}{p}} d A\right)^{\frac{p}{p^{\prime}}}<\infty .
$$

If $z_{0} \in \mathbb{C}$ and $\gamma \in \mathbb{R}, \omega(z)=\left(1+\left|z+z_{0}\right|\right)^{\gamma}$ and $\omega(z)=e^{\gamma\left|z+z_{0}\right|}$ belong to $\cup_{p>1} A_{p}^{\text {restricted }}$ (see the proof of Lemma 2.1 below).

We recall that for a measurable function $f$ in $\mathbb{C}$ the Berezin transform of $f$ is given by

$$
\widetilde{f}^{(\alpha)}(z):=\frac{\alpha}{\pi} \int_{\mathbb{C}} e^{-\alpha|z-u|^{2}} f(u) d A(u) .
$$

Theorem A. Let $\beta>0,1<p<\infty$ and $\omega$ a weight such that $\omega(z)>0$ a. e. on $\mathbb{C}$. Then, the following conditions are equivalent;

(i) $\omega$ belongs to $A_{p, r}$ for some $r>0$;

(ii) $\omega$ belongs to $A_{p, r}$ for any $r>0$;

(iii) For each $\alpha, \gamma>0$

$$
\sup _{z \in \mathbb{C}} \widetilde{\omega}^{(\alpha)}(z)\left(\widetilde{\omega^{-\frac{p^{\prime}}{p}}}(\gamma)\right)^{\frac{p}{p^{\prime}}}<\infty
$$

(iv) $P_{\beta}$ is bounded on $\mathcal{L}_{\beta, \omega}^{p}$;

(v) $P_{\beta}^{+}$is bounded on $\mathcal{L}_{\beta, \omega}^{p}$.

The conditions (iii) and (v) in Theorem A do not appear in the statement of [10, Theorem 3.1]. The equivalence of (v) with (ii) follows easily from the proof of [10, Theorem 3.1]. Moreover, the "invariant" description of $A_{p, r}$ 
weights obtained via the Berezin transform was proved in [10, p. 397] (it also follows from Lemma 2.8 below).

In order to complete the picture about the boundedness of the Bergman projection on $\mathcal{L}_{\beta, \omega}^{p}$, we will deal with the remaining case $p=1$. In particular, fixed $r>0$, we say that a weight $\omega \in A_{1, r}$ if $\omega(z)>0$ a.e. on $\mathbb{C}$ and there is a constant $C=C(r, \omega)$ such that for any square with $l(Q)=r$,

$$
\frac{\omega(Q)}{|Q|} \leq C \omega(u), \quad \text { a. e. } u \in Q .
$$

We denote

$$
\mathcal{C}_{1, r}(\omega):=\sup _{Q, l(Q)=r} \frac{\omega(Q)}{|Q| \operatorname{infess}_{u \in Q} \omega(u)} .
$$

We will prove an analogous version of Theorem $\mathrm{A}$ for $A_{1, r}$ weights (see Proposition 2.7 below).

In view of Theorem $\mathrm{A}$ and Proposition 2.7, the condition $A_{p, r}$ does not depend on $r$. So from now on, following the notation in [10], we will write $A_{p}^{\text {restricted }}$ for these classes of weights. Moreover, $\omega \in A_{p}^{\text {restricted }}$ if and only if $P_{\alpha}^{+}$is bounded on $\mathcal{L}_{\alpha, \omega}^{p}$ for some (equivalently for any) $\alpha>0$.

Similarly to Muckenhoupt weights, we denote

$$
A_{\infty}^{\text {restricted }}:=\bigcup_{1 \leq q<\infty} A_{q}^{\text {restricted }} .
$$

A discussion about properties and examples of $A_{p}^{\text {restricted-weights, as well as }}$ similarities and differences with the classical Muckenhoupt weights, will be provided in Section 2. In particular, we sketch the proof of a character-

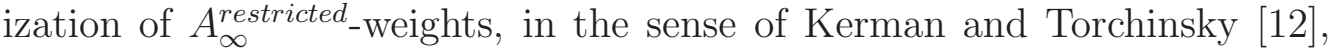
obtained in [7].

Equipping a space of functions with different equivalent norms results to be quite effective in the study of function and concrete operator theory $[1,5,17,20,21]$. In the context of Fock spaces, it is known that (see $[3,4,5])$ for any $\alpha, p>0$

$$
\|f\|_{\mathcal{F}_{\alpha}^{p}}^{p} \asymp \sum_{j=0}^{k-1}\left|f^{(j)}(0)\right|^{p}+\int_{\mathbb{C}} \frac{\left|f^{(k)}(z)\right|^{p}}{(1+|z|)^{k p}} e^{-p \frac{\alpha}{2}|z|^{2}} d A(z), \quad f \in H(\mathbb{C}),
$$

that is, the distortion function in the above Littlewood-Paley formula is $\psi(z)=\frac{1}{1+|z|}$. We will use Theorem A, among other techniques, to prove an extension of (1.1) to weighted Fock spaces induced by $A_{\infty}^{\text {restricted }}$ - weights.

Theorem 1.1. Let $\omega \in A_{\infty}^{\text {restricted }}$. Then, for $0<p<\infty, k \in \mathbb{N}$ and $\alpha>0$ $\|f\|_{\mathcal{F}_{\alpha, \omega}^{p}}^{p} \asymp \sum_{j=0}^{k-1}\left|f^{(j)}(0)\right|^{p}+\int_{\mathbb{C}}\left|f^{(k)}(z)\right|^{p} e^{-p \frac{\alpha}{2}|z|^{2}} \frac{\omega(z)}{(1+|z|)^{k p}} d A(z), \quad f \in H(\mathbb{C})$. 
As for the proof of Theorem 1.1, it is enough to prove the case $k=1$ because

$$
\omega_{\gamma}(z):=\frac{\omega(z)}{(1+|z|)^{\gamma}} \in A_{\infty}^{\text {restricted }}, \quad \gamma \in \mathbb{R}
$$

whenever $\omega \in A_{\infty}^{\text {restricted }}$ (see Lemma 2.1 below).

Later on, we apply Theorem 1.1 to study the boundedness of the differentiation and integration operator. We denote $D^{(n)}(f)=f^{(n)}, n \in \mathbb{N}$, $D^{(0)}(f)=f, D^{(-1)}(f)(z)=\int_{0}^{z} f(\zeta) d \zeta$ and $D^{(-n)}(f)=D^{(-n+1)} \circ D^{(-1)}(f)$, $n \in \mathbb{N}$. Given $0<\alpha, p, q<\infty$, a positive Borel measure $\mu$ on $\mathbb{C}$ is a $q$ Carleson measure for $\mathcal{F}_{\alpha, \omega}^{p}$ if the identity $I_{d}: \mathcal{F}_{\alpha, \omega}^{p} \rightarrow L^{q}(\mu)$ is a bounded operator. We denote by $D(z, r)$ the Euclidean disc of center $z$ and radius $r>0$.

Theorem 1.2. Let $\alpha \in(0, \infty), n \in \mathbb{Z}, \omega \in A_{\infty}^{\text {restricted }}$ and $\mu$ a finite positive Borel measure on $\mathbb{C}$ such that $\sup _{k \in \mathbb{N}}\left\|z^{k}\right\|_{L^{q}(\mu)}<\infty$.

If $0<p \leq q<\infty$, the following conditions are equivalent:

(i) $D^{(n)}: \mathcal{F}_{\alpha, \omega}^{p} \rightarrow L^{q}(\mu)$ is a bounded operator;

(ii) $\mu$ is a q-Carleson measure for $\mathcal{F}_{\alpha, \omega_{n p}}^{p}$;

(iii) The function

$$
G(a):=\frac{1}{\left(\omega_{n p}(D(a, 1))\right)^{\frac{q}{p}}} \int_{D(a, 1)} e^{\alpha \frac{q}{2}|z|^{2}} d \mu(z)
$$

is bounded on $\mathbb{C}$.

Moreover, if $n \in \mathbb{N} \cup\{0\}$

$$
\left\|I_{d}\right\|_{\mathcal{F}_{\alpha, \omega_{n p}}^{p} \rightarrow L^{q}(\mu)}^{q} \asymp\left\|D^{(n)}\right\|_{\mathcal{F}_{\alpha, \omega}^{p} \rightarrow L^{q}(\mu)}^{q} \asymp\|G\|_{L^{\infty}},
$$

and for $n$ a negative integer,

$$
\left\|I_{d}\right\|_{\mathcal{F}_{\alpha, \omega_{n} p}^{p} \rightarrow L^{q}(\mu)}^{q}+C_{\mu, n} \asymp\left\|D^{(n)}\right\|_{\mathcal{F}_{\alpha, \omega}^{p} \rightarrow L^{q}(\mu)}^{q}+C_{\mu, n} \asymp\|G\|_{L^{\infty}}+C_{\mu, n} .
$$

where $C_{\mu, n}=\max _{k=0, \ldots,-n-1}\left\|z^{k}\right\|_{L^{q}(\mu)}^{q}<\infty$.

If $0<q<p<\infty$, the following conditions are equivalent:

(i) $D^{(n)}: \mathcal{F}_{\alpha, \omega}^{p} \rightarrow L^{q}(\mu)$ is a bounded operator;

(ii) $\mu$ is a q-Carleson measure for $\mathcal{F}_{\alpha, \omega_{n p}}^{p}$;

(iii) The function

$$
H(u):=\frac{1}{\omega_{n p}(D(u, 1))} \int_{D(u, 1)} e^{\frac{q \alpha|z|^{2}}{2}} d \mu(z)
$$

is in $L^{\frac{p}{p-q}}\left(\mathbb{C}, \omega_{n p}\right)$.

Moreover, if $n \in \mathbb{N} \cup\{0\}$,

$$
\left\|I_{d}\right\|_{\mathcal{F}_{\alpha, \omega_{n p}}^{p} \rightarrow L^{q}(\mu)}^{q} \asymp\left\|D^{(n)}\right\|_{\mathcal{F}_{\alpha, \omega}^{p} \rightarrow L^{q}(\mu)}^{q} \asymp\|H\|_{L^{\frac{p}{p-q}}\left(\mathbb{C}, \omega_{n p}\right)},
$$


and if $n$ is a negative integer,

$$
\left\|I_{d}\right\|_{\mathcal{F}_{\alpha, \omega_{n p}}^{p} \rightarrow L^{q}(\mu)}^{q}+C_{\mu, n} \asymp\left\|D^{(n)}\right\|_{\mathcal{F}_{\alpha, \omega}^{p} \rightarrow L^{q}(\mu)}^{q}+C_{\mu, n} \asymp\|H\|_{L^{\frac{p}{p-q}}\left(\mathbb{C}, \omega_{n p}\right)}+C_{\mu, n} .
$$

It is worth noticing that the technical assumption $\sup _{k \in \mathbb{N}}\left\|z^{k}\right\|_{L^{q}(\mu)}<\infty$ in Theorem 1.2, which is only used when $n$ is a negative integer, is not a real restriction (see Lemma 2.8 below). Let us also consider the Fock-Sobolev spaces $\mathcal{F}_{\alpha, \omega}^{p, n}, n \in \mathbb{N}$, of the entire functions such that

$$
\|f\|_{\mathcal{F}_{\alpha, \omega}^{p, n}}^{p}:=\sum_{k=0}^{n-1}\left|f^{(k)}(0)\right|^{p}+\int_{\mathbb{C}}\left|f^{(n)}(z)\right|^{p} e^{-p \frac{\alpha}{2}|z|^{2}} \omega(z) d A(z)<\infty .
$$

By Theorem 1.1 and Lemma 2.1 (below), $\mathcal{F}_{\alpha, \omega}^{p, n}$ coincides with $\mathcal{F}_{\alpha, \omega_{-n p}}^{p}$, so Theorem 1.2 also gives a description of the $q$-Carleson measures for Fock-Sobolev spaces induced by $A_{\infty}^{\text {restricted }}$-weights. A characterization of $q$-Carleson measures for classical Fock-Sobolev spaces is provided in [15], see also $[3,11]$.

As a consequence of Theorem 1.2 we obtain characterizations of pointwise multipliers between weighted Fock spaces, $\mathcal{F}_{\alpha, \omega}^{p}$ and $\mathcal{F}_{\beta, \eta}^{q}$, for $0<q, p<\infty$, $\eta$ a weight and $\omega \in A_{\infty}^{\text {restricted }}$, which are particularly neat for $\omega=\eta$, see Theorem 5.4 below.

This paper is organized as follows. In Section 2 we study $A_{p}^{\text {restricted-weights }}$ and prove a collection of preliminary results which will be employed to prove Proposition 2.7. These estimates will also be used to prove Theorem 1.1 in Section 3. We prove Theorem 1.2 in Section 4. Finally, in Section 5 we apply the descriptions on Carleson measures for $\mathcal{F}_{\alpha, \omega}^{p}$ to obtain characterizations of pointwise multipliers between weighted Fock spaces.

For two real-valued functions $E_{1}, E_{2}$ we write $E_{1} \asymp E_{2}$, or $E_{1} \lesssim E_{2}$, if there exists a positive constant $k$ independent of the argument such that $\frac{1}{k} E_{1} \leq E_{2} \leq k E_{1}$, respectively $E_{1} \leq k E_{2}$. Given a weight $\omega \in A_{p}^{\text {restricted }}$, $1<p<\infty$, we denote the conjugate weight $\omega^{-p^{\prime} / p}$ by $\omega^{\prime}$. We write $\mathcal{R}(z)$ for the real part of a complex number $z$.

\section{2. $A_{p}^{\text {restricted }}$ Weights versus Classical Muckenhoupt $A_{p}$ Weights.}

In this section we briefly discuss some aspects of the theory of $A_{p}^{\text {restricted }}$ weights, in particular we give examples and descriptions of these classes of weights. Some of these characterizations are not used in the proofs of further results in this work. However we consider relevant to understand the basic features of $A_{p}^{\text {restricted }}$-weights, specially comparing them with their analogues into the theory of classical Muckenhoupt weights. We recall that $\omega$ belongs to the class of Muckenhoupt weights $A_{p}, 1<p<\infty$, if

$$
A_{p}(\omega):=\sup _{Q} \frac{\omega(Q)}{|Q|}\left(\frac{\omega^{\prime}(Q)}{|Q|}\right)^{\frac{p}{p^{\prime}}}<\infty
$$


where the supremum runs over all the squares $Q \subset \mathbb{C}$. In addition, $\omega \in A_{1}$ if

$$
A_{1}(\omega):=\sup _{Q} \frac{\omega(Q)}{|Q| \operatorname{infess}_{u \in Q} \omega(u)}<\infty .
$$

An extensive study of these weights can be found, for instance, in $[9,18]$.

2.1. The class $A_{p}^{\text {restricted }}$ with $1 \leq p<\infty$.

We begin gathering some properties of $A_{p}^{\text {restricted }}$-weights which are analogous to the properties of Muckenhoupt weights (see [18, p. 195]). We write $Q_{r}(z)$ for the square of center $z \in \mathbb{C}$, with sides parallel to the coordinate axes and $l(Q)=r$.

Lemma 2.1. Let $p \geq 1, \gamma \in \mathbb{R}$ and $\omega \in A_{p}^{\text {restricted. Then }}$

$$
\omega_{\gamma}=\frac{\omega(z)}{(1+|z|)^{\gamma}} \in A_{p}^{\text {restricted }} .
$$

Proof. Fix $r>0$. Then for any $z_{0} \in \mathbb{C}$,

$$
\frac{(1+|z|)}{1+r} \leq\left(1+\left|z_{0}\right|\right) \leq(1+r)(1+|z|), \quad z \in Q_{r}\left(z_{0}\right) .
$$

So, it follows that $\frac{\omega(z)}{(1+|z|)^{\gamma}} \in A_{p}^{\text {restricted }}$.

Proposition 2.2. Let $\omega$ be a weight such that $\omega(z)>0$ a. e. on $\mathbb{C}$. Then,

(i) If $1<p<\infty, \omega \in A_{p}^{\text {restricted }}$ if and only if $\omega^{\prime}=\omega^{-p^{\prime} / p} \in A_{p^{\prime}}^{\text {restricted }}$.

(ii) If $1 \leq p<\infty, \omega \in A_{p}^{\text {restricted }}$ if and only if for some (equivalently for any) $r>0$ there is a constant $C_{r}>0$ such that for any nonnegative measurable function $f$

$\left(\frac{1}{|Q|} \int_{Q} f d A\right)^{p} \leq \frac{C_{r}}{\omega(Q)} \int_{Q} f^{p} \omega d A, \quad$ for any $Q$ with $l(Q)=r$.

(iii) If $1 \leq p<q<\infty, A_{p}^{\text {restricted }} \subsetneq A_{q}^{\text {restricted }}$.

(iv) If $1 \leq p<\infty, A_{p} \subsetneq A_{p}^{\text {restricted }}$.

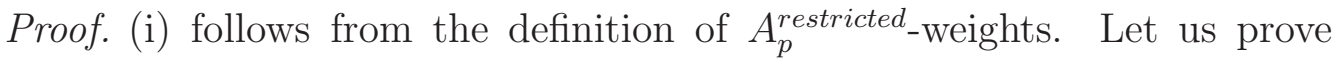
(ii). If $p>1$, Hölder's inequality gives that any $\omega \in A_{p}^{\text {restricted }}$ satisfies (2.1). Conversely if a weight satisfies (2.1), by choosing $f=(\omega+\varepsilon)^{-p^{\prime} / p}$, $\varepsilon>0$, and taking limit $\varepsilon \rightarrow 0^{+}$, we deduce that $\omega \in A_{p}^{\text {restricted }}$. Now, if $\omega \in A_{1}^{\text {restricted }}$, then for any $f \geq 0$, and any square $Q$,

$$
\frac{1}{|Q|} \int_{Q} f d A \leq \frac{C_{r}}{\omega(Q)} \int_{Q} f \omega d A .
$$

On the other hand, if $\omega$ satisfies (2.2) then Hölder's inequality gives that for any $1<p<\infty$,

$$
\left(\frac{1}{|Q|} \int_{Q} f d A\right)^{p} \leq \frac{C_{r}^{p}}{\omega(Q)} \int_{Q} f^{p} \omega d A
$$


which implies that $\omega \in A_{p}^{\text {restricted }}$ and $\mathcal{C}_{p, r}(\omega) \leq C_{r}^{p}$. Since

$$
\lim _{p \rightarrow 1^{+}}\left(\frac{1}{|Q|} \int_{Q} \omega^{-\frac{p^{\prime}}{p}} d A\right)^{\frac{p}{p^{\prime}}}=\operatorname{supess}_{u \in Q} \omega^{-1}(u),
$$

we conclude that $\omega \in A_{1}^{\text {restricted }}$.

(iii) for $p>1$ is a consequence of Hölder's inequality, where $\omega(z)=$ $|z|^{2(\delta-1)}$, for $\delta \in(p, q)$ proves that the inclusion is strict. If $\omega \in A_{1}^{\text {restricted }}$, the assertion follows from the fact that (2.3) holds for any $1<p<\infty$.

Finally, it is clear that $A_{p} \subset A_{p}^{\text {restricted }}$. In order to see that the embedding is strict, one can choose the weight $\omega(z)=\left(1+|z|^{2}\right)^{p-1}$, for $p>1$. In fact, Lemma 2.1 shows that $\omega \in A_{p}^{\text {restricted }}$ and since

$$
\begin{aligned}
& \frac{1}{|D(0, r)|} \int_{D(0, r)} \omega d A\left(\frac{1}{|D(0, r)|} \int_{D(0, r)} \omega^{-p^{\prime} / p} d A\right)^{\frac{p}{p^{\prime}}} \\
& \asymp\left(\log \frac{1}{1+r^{2}}\right)^{p-1} \rightarrow \infty, \quad r \rightarrow \infty
\end{aligned}
$$

$\omega$ is not a classical $A_{p}$-Muckenhoupt weight. As for $p=1, \omega(z)=e^{|z|} \in$ $A_{1}^{\text {restricted }} \backslash A_{1}$.

The following lemma is proved in [10, Lemma 3.4].

Lemma B. For each $r>0$, let $r \mathbb{Z}^{2}$ denote the set $\left\{r k_{1}+\right.$ ir $\left.k_{2}: k_{1}, k_{2} \in \mathbb{Z}\right\}$. If $\omega \in A_{p}^{\text {restricted }}$, then there is a constant $M=M(\omega, r, p)$ such that

$$
\frac{\omega\left(Q_{r}(\nu)\right)}{\omega\left(Q_{r}\left(\nu^{\prime}\right)\right)} \leq M^{\left|\nu-\nu^{\prime}\right|}
$$

for all $\nu, \nu^{\prime} \in r \mathbb{Z}^{2}$.

Remark 2.3. The above lemma shows that for each $L>0, \omega\left(Q_{r}(\nu)\right) \asymp$ $\omega\left(Q_{r}\left(\nu^{\prime}\right)\right)$ if $\left|\nu-\nu^{\prime}\right|<L$ and $\omega \in A_{\infty}^{\text {restricted }}$. So, fixed $N>r$ we have that

$$
\omega\left(Q_{r}(\nu)\right) \asymp \omega\left(Q_{N r}\left(\nu^{\prime}\right)\right) .
$$

Combining these results it follows that

$$
\omega\left(Q_{r}(z)\right) \asymp \omega\left(Q_{R}(w)\right), \quad \text { for any } z, w \text { such that }|z-w|<L,
$$

whenever $\omega \in A_{\infty}^{\text {restricted }}, r<L$ and $R \in(r, N r)$.

In particular, if $\omega \in A_{\infty}^{\text {restricted }}$, for any $t>0$ and $N \in \mathbb{N}$ there is $C=$ $C(\omega, N, p)$ such that

$$
\omega(D(a, t)) \leq \omega(D(a, N t)) \leq C \omega(D(a, t)), \quad a \in \mathbb{C} .
$$

As a consequence, squares of fixed length can be replaced by discs of fixed radius in the study of $A_{p}^{\text {restricted }}$ weights.

Now, let us observe that the class $A_{p}^{\text {restricted }}$ is invariant under translations. 
Lemma 2.4. Let $p \in[1, \infty)$. Then, a weight $\omega$ belongs to $A_{p}^{\text {restricted }}$ if and only for any (equivalently for some) $a \in \mathbb{C}, \omega_{[a]}(u):=\omega(a+u) \in A_{p}^{\text {restricted }}$. Moreover,

$$
\mathcal{C}_{p, r}(\omega)=\mathcal{C}_{p, r}\left(\omega_{[a]}\right)
$$

Proof. The proof follows immediately from the invariance under translations of the Lebesgue measure and the definition of the $A_{p}^{\text {restricted-weights. }}$

A fundamental property of the classical Muckenhoupt weights is the reverse Hölder inequality [6, Theorem 7.4]. So, it is natural to ask whether or not for each $A_{p}^{\text {restricted }}$-weight $\omega$ there exists $\varepsilon>0$ and $C(\varepsilon, r)>0$ such that

$$
\left(\frac{1}{|Q|} \int_{Q} \omega^{1+\varepsilon}(\zeta) d A(\zeta)\right)^{\frac{1}{1+\varepsilon}} \leq C(\varepsilon, r) \frac{1}{|Q|} \int_{Q} \omega(\zeta) d A(\zeta)
$$

for every cube $Q$ with $l(Q)=r$. The following considerations provide a negative answer to this question.

Remark 2.5. - The weight $\omega(z)=|z|^{-2}\left(\log \frac{1}{|z|}\right)^{-2} \in A_{2}^{\text {restricted }}$, but for each $\varepsilon>0, \omega^{1+\varepsilon} \notin A_{2}^{\text {restricted }}$. So $\omega$ does not satisfy (2.6).

- The weight

$$
v(z)=|z|^{2}\left(\log \frac{1}{|z|}\right)^{2}
$$

is in $A_{2}^{\text {restricted }} \backslash \cup_{1<p<2} A_{p}^{\text {restricted }}$. That is, the $A_{p}^{\text {restricted }}$-weights do not satisfy the natural analogue of the $(p-\varepsilon)$-condition which holds for the classical $A_{p}$-Muckenhoupt weights. Moreover, sincee

$$
\begin{aligned}
& \frac{1}{|D(0, r)|^{2}}\left(\int_{D(0, r)} v(z) d A(z)\right)\left(\int_{D(0, r)} v^{-1}(z) d A(z)\right) \\
& \asymp \log \frac{1}{r} \rightarrow \infty, \quad r \rightarrow 0,
\end{aligned}
$$

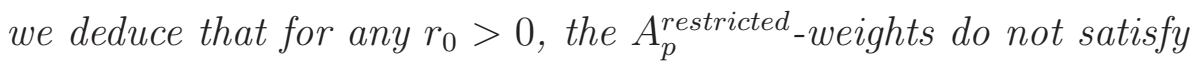
the uniform condition

$$
\sup _{Q_{r}, r \leq r_{0}} \frac{1}{r^{2 p}} \int_{Q_{r}} \omega d A\left(\int_{Q_{r}} \omega^{-\frac{p^{\prime}}{p}} d A\right)^{\frac{p}{p^{\prime}}}<\infty .
$$

\subsection{The class $A_{\infty}^{\text {restricted }}$.}

In the classical setting, there are a good number of equivalent conditions which describe the class $A_{\infty}=\bigcup_{1 \leq q<\infty} A_{q}$ (see [7], [18, Chapter 5] or [6, p. 149]). So, it is natural to ask whether or not the class $\bigcup_{q \geq 1} A_{q}^{\text {restricted }}$ can be described by neat analogous conditions to those describing the class $A_{\infty}$. With this aim, we introduce the following class of weights. 
Definition 2.6. A weight $\omega$ satisfies the $K T_{r}$-property, $r>0$, if there exist constants $r>0, \delta \in(0,1)$ and $C_{r}>0$ such that for any square $Q$ with $l(Q)=r$ and every measurable set $E \subset Q$ it holds that

$$
\frac{|E|}{|Q|} \leq C_{r}\left(\frac{\omega(E)}{\omega(Q)}\right)^{\delta}
$$

If we replace in (2.7) the constant $C_{r}$ by an absolute constant $C$ and $Q$ runs over all the squares $Q$, it is obtained a condition which describes the class $A_{\infty}$ of Muckenhoupt weights [7, Theorem 3.1]. It was introduced by Kerman and Torchinsky in [12, Proposition 1] in order to describe the restricted weak-type for the Hardy-Littlewood maximal operator. It follows from [7, Theorem 3.1] (a result which holds for general basis) that, for each $r>0$, a weight $\omega \in A_{\infty}^{\text {restricted }}$ if and only if it satisfies the $K T_{r}$-property. For the sake of completeness we offer a direct short proof based on the ideas of the proof of [7, Thorem 3.1].

Proposition C. Let $\omega$ be a weight. Then, the following conditions are equivalent:

(i) $\omega \in A_{\infty}^{\text {restricted }}$;

(ii) $\omega$ satisfies the Kerman-Torchinsky $K T_{r}$-property for any $r>0$;

(iii) $\omega$ satisfies the Kerman-Torchinsky $K T_{r}$-property for some $r>0$.

Proof. (i) $\Rightarrow$ (ii). Fix $r>0$ and take $p>1$ such that $\omega \in A_{p, r}$. Then, if $l(Q)=r$ and $E \subset Q$, then

$$
\begin{aligned}
|E| & \leq \omega(E)^{1 / p}\left(\omega^{\prime}(E)\right)^{1 / p^{\prime}} \leq \omega(E)^{1 / p}\left(\omega^{\prime}(Q)\right)^{1 / p^{\prime}} \\
& \leq \mathcal{C}_{p, r}^{1 / p}(\omega) \omega(E)^{1 / p}|Q|^{1 / p^{\prime}}\left(\frac{|Q|}{\omega(Q)}\right)^{1 / p}
\end{aligned}
$$

that is $\frac{|E|}{|Q|} \leq \mathcal{C}_{p, r}^{1 / p}(\omega)\left(\frac{\omega(E)}{\omega(Q)}\right)^{1 / p}$, which gives (ii) with $\delta=\frac{1}{p}$.

(iii) $\Rightarrow$ (i). Let $Q$ be a square with $l(Q)=r$. For each $\lambda>0$, let us denote $E_{\lambda}=\left\{z \in Q: \omega(z)<\frac{1}{\lambda}\right\}$. Then, by hypothesis there is $\delta \in(0,1)$ and $C_{r}>0$

$$
\lambda \omega\left(E_{\lambda}\right) \leq\left|E_{\lambda}\right| \leq C_{r}|Q|\left(\frac{\omega\left(E_{\lambda}\right)}{\omega(Q)}\right)^{\delta},
$$

that is

$$
\lambda^{\frac{1}{1-\delta}} \omega\left(E_{\lambda}\right) \leq C_{r, \delta}\left(\frac{|Q|}{\omega(Q)^{\delta}}\right)^{\frac{1}{1-\delta}}
$$


Take $p$ such that $p^{\prime} \in\left(1, \frac{1}{1-\delta}\right)$. Then, if we denote $d \omega=\omega d A$, for any $M>0$

$$
\begin{aligned}
\int_{Q} \omega^{-p^{\prime} / p} d A & =\int_{Q} \omega^{-p^{\prime}} d \omega=p^{\prime} \int_{0}^{\infty} \lambda^{p^{\prime}-1} \omega\left(E_{\lambda}\right) d \lambda \\
& \leq \omega(Q) p^{\prime} \int_{0}^{M} \lambda^{p^{\prime}-1} d \lambda+C_{r, \delta}\left(\frac{|Q|}{\omega(Q)^{\delta}}\right)^{\frac{1}{1-\delta}} \int_{M}^{\infty} \lambda^{p^{\prime}-1-\frac{1}{1-\delta}} d \lambda \\
& =\omega(Q) M^{p^{\prime}}+C_{r, p, \delta}\left(\frac{|Q|}{\omega(Q)^{\delta}}\right)^{\frac{1}{1-\delta}} M^{p^{\prime}-\frac{1}{1-\delta}}
\end{aligned}
$$

so choosing $M=\frac{|Q|}{\omega(Q)}$ we get

$$
\frac{\int_{Q} \omega^{-p^{\prime}+1} d A}{|Q|} \leq C_{r, p, \delta}\left(\frac{|Q|}{\omega(Q)}\right)^{p^{\prime}-1},
$$

that is $\omega \in A_{p, r}$, which together with Theorem A finishes the proof.

2.3. The class $A_{1}^{\text {restricted }}$. The primary aim of this section consists on proving the following result.

Proposition 2.7. Let $\beta>0$ and $\omega$ be a weight such that $\omega(z)>0$ a.e. on $\mathbb{C}$. Then, the following conditions are equivalent;

(i) $\omega$ belongs to $A_{1, r}$ for some $r>0$;

(ii) $\omega$ belongs to $A_{1, r}$ for any $r>0$;

(iii) For any $\alpha>0$ there is a positive constant $C$ such that

$$
\widetilde{\omega}^{(\alpha)}(z) \leq C \omega(z) \quad \text { a.e. } z \in \mathbb{C} ;
$$

(iv) $P_{\beta}$ is bounded on $\mathcal{L}_{\beta, \omega}^{1}$;

(v) $P_{\beta}^{+}$is bounded on $\mathcal{L}_{\beta, \omega}^{1}$.

Some preliminary results, which will also be used to prove Theorem 1.1, are needed.

Lemma 2.8. Assume that $\omega \in A_{\infty}^{\text {restricted }}$. Then, there exists $r_{0}$ such that for any $r \in\left(0, r_{0}\right)$ and for any $\beta>0$

$$
\int_{\mathbb{C}} e^{-\beta|z|^{2}} \omega(z) d A(z) \leq C(\omega, r, \beta) \omega\left(Q_{r}(0)\right)<\infty
$$

Proof. Choose $r_{0}>0$ such that $|z-\nu| \leq 1$ if $z \in Q_{r_{0}}(\nu)$. So

$$
|\nu| \leq 1+|z|, \quad z \in Q_{r}(\nu), r \in\left(0, r_{0}\right) .
$$

Given $\beta>0$, choose $\alpha \in(0, \beta)$ and $R=R(\beta)$ such that

$$
\alpha|z|^{2}+2 \alpha|z| \leq \beta|z|^{2}, \quad \text { if }|z| \geq R .
$$

By (2.5) it is enough to prove that

$$
\int_{\mathbb{C}} e^{-\alpha|z|^{2}-2 \alpha|z|} \omega(z) d A(z) \leq C(\alpha, r, \omega) \omega\left(Q_{r}(0)\right)<\infty .
$$


Now, bearing in mind (2.8) and Lemma B we deduce that

$$
\begin{aligned}
\int_{\mathbb{C}} e^{-\alpha|z|^{2}-2 \alpha|z|} \omega(z) d A(z) & =\sum_{\nu \in r \mathbb{Z}^{2}} \int_{Q_{r}(\nu)} e^{-\alpha|z|^{2}-2 \alpha|z|} \omega(z) d A(z) \\
& \leq e^{\alpha} \sum_{\nu \in r \mathbb{Z}^{2}} e^{-\alpha|\nu|^{2}} \int_{Q_{r}(\nu)} \omega(z) d A(z) \\
& \leq C(\alpha, r, \omega) \omega\left(Q_{r}(0)\right) \sum_{\nu \in r \mathbb{Z}^{2}} e^{-\alpha|\nu|^{2}} M^{|\nu|} \\
& \leq C(\alpha, r, \omega) \omega\left(Q_{r}(0)\right)<\infty .
\end{aligned}
$$

This finishes the proof.

Lemma 2.9. If $\omega \in A_{\infty}^{\text {restricted }}$, then for any $\beta, t, r>0$ and $\gamma \in \mathbb{R}$

$$
\int_{\mathbb{C}} e^{-\beta|u|^{2}} \omega(D(u, t))^{\gamma} d A(u) \leq C(\gamma, \beta, r, \omega, t) \omega\left(Q_{r}(0)\right)^{\gamma}<\infty .
$$

Proof. Fixed $\beta>0$, choose $r_{0}$ and $\alpha$ as in the proof of Lemma 2.8. It is enough to prove that

$$
\int_{\mathbb{C}} e^{-\alpha|u|^{2}-2 \alpha|u|} \omega(D(u, t))^{\gamma} d A(u) \leq C(\alpha, r, \omega, \gamma, t) \omega\left(Q_{r}(0)\right)^{\gamma}<\infty .
$$

Next, take $N=N(t, r) \in \mathbb{N}$ such that

$$
D(u, t) \subset Q_{N}(\nu), \quad u \in Q_{r}(\nu), \quad \nu \in r \mathbb{Z}^{2} .
$$

Now, bearing in mind (2.8), (2.9), Lemma B and (2.5), we deduce that

$$
\begin{aligned}
\int_{\mathbb{C}} e^{-\alpha|u|^{2}-2 \alpha|u|} \omega & (D(u, t))^{\gamma} d A(u) \\
\leq & e^{\alpha} \sum_{\nu \in r \mathbb{Z}^{2}} e^{-\alpha|\nu|^{2}} \int_{Q_{r}(\nu)} \omega(D(u, t))^{\gamma} d A(u) \\
\leq & r^{2} e^{\alpha} \sum_{\nu \in r \mathbb{Z}^{2}} e^{-\alpha|\nu|^{2}} \omega\left(Q_{N}(\nu)\right)^{\gamma} \\
\leq & C(\alpha, r) \sum_{\nu \in r \mathbb{Z}^{2}} e^{-\alpha|\nu|^{2}} M^{\gamma|\nu|} \omega\left(Q_{N}(0)\right)^{\gamma} \\
& \leq C(\alpha, r, \omega, t) \omega\left(Q_{r}(0)\right)^{\gamma} \sum_{\nu \in r \mathbb{Z}^{2}} e^{-\alpha|\nu|^{2}} M^{\gamma|\nu|} \\
& \leq C(\alpha, r, \omega, \gamma, t) \omega\left(Q_{r}(0)\right)^{\gamma}<\infty
\end{aligned}
$$

Next assume that $\gamma<0$. Take $p \in(1, \infty)$ such that $\omega \in A_{p}^{\text {restricted }}$. Then, $\omega^{\prime} \in A_{\infty}^{\text {restricted }}$ and

$$
\omega(D(u, t))^{\gamma} \asymp \omega^{\prime}(D(u, t))^{-p \gamma / p^{\prime}}, \quad u \in \mathbb{C},
$$

so the result follows by applying the above argument to $\omega^{\prime}$. This finishes the proof. 
2.4. Proof of Proposition 2.7. (iii) $\Rightarrow$ (ii). Take $Q$ with $l(Q)=r>0$. Then for any $z, \zeta \in Q,|z-\zeta|^{2} \leq 2 r^{2}$, so

$$
e^{-2 \alpha r^{2}} \omega(Q) \leq C(\alpha) \widetilde{\omega}^{(\alpha)}(z) \leq C(\alpha, \omega) \omega(z), \quad \text { a.e. } z \in Q
$$

which implies (ii) .

(i) $\Rightarrow$ (iii). Fixed $\alpha>0, r>0$ such that $\omega \in A_{1, r}$ and $z \in \mathbb{C}$. Then, by Lemma 2.4 and Lemma 2.8

$$
\begin{aligned}
\widetilde{\omega}^{(\alpha)}(z) & =\int_{\mathbb{C}} e^{-\alpha|\zeta|^{2}} \omega(\zeta+z) d A(\zeta) \leq C(\alpha, r, \omega) \omega_{[z]}\left(Q_{r}(0)\right) \\
& =C(\alpha, r, \omega) \omega\left(Q_{r}(z)\right) \\
& \leq C(\alpha, r, \omega) \operatorname{infess}_{u \in Q_{r}(z)} \omega(u) \leq \omega(z), \quad \text { a.e. } z \in \mathbb{C} .
\end{aligned}
$$

Therefore, we already have proved (i) $\Leftrightarrow$ (ii) $\Leftrightarrow$ (iii) .

Now, let us observe that $\left(\mathcal{L}_{\beta}^{1}(\omega)\right)^{\star} \simeq L^{\infty}$ via the pairing

$$
\langle f, g\rangle_{\mathcal{L}_{\beta / 2}^{2}(\omega)}=\int_{\mathbb{C}} f(z) \overline{g(z)} e^{-\frac{\beta}{2}|z|^{2}} \omega(z) d A(z) .
$$

So, bearing in mind that the adjoint of $P_{\beta}$ (via the $\mathcal{L}_{\beta / 2}^{2}(\omega)$-pairing) is

$$
P_{\beta}^{\star}(f)(z)=\frac{\beta}{\pi \omega(z)} \int_{\mathbb{C}} f(\zeta) e^{-\frac{\beta}{2}|\zeta-z|^{2}+i \beta \operatorname{Im}(z \bar{\zeta})} \omega(\zeta) d A(\zeta),
$$

we deduce that (iv) holds if and only if

$$
\operatorname{supesss}_{z \in \mathbb{C}}\left|\frac{1}{\omega(z)} \int_{\mathbb{C}} f(\zeta) e^{-\frac{\beta}{2}|\zeta-z|^{2}+i \beta \operatorname{Im}(z \bar{\zeta})} \omega(\zeta) d A(\zeta)\right| \leq C\|f\|_{L^{\infty}} .
$$

Therefore, it is clear that (iii) $\Rightarrow($ iv). In order to see the reverse implication, for each $z \in \mathbb{C}$ choose $f_{z}(\zeta)=e^{-i \beta \operatorname{Im}(z \bar{\zeta})}$. The equivalence (iii) $\Leftrightarrow(\mathrm{v})$ can be proved in the same way. This finishes the proof.

\section{A Littlewood-Paley formula fOR $\mathcal{F}_{\alpha, \omega}^{p}$.}

\subsection{Preliminary results.}

In this section we will prove a collection of estimates which will be essential to prove Theorem 1.1.

Lemma 3.1. Let $0<p, t<\infty, \alpha \geq 0$ and $\omega \in A_{\infty}^{\text {restricted }}$. Then, there exists a constant $C=C(\alpha, p, \omega, t)$ such that

$$
|f(z)|^{p} e^{-\frac{p \alpha|z|^{2}}{2}} \leq \frac{C}{\omega(D(z, t))} \int_{D(z, t)}|f(u)|^{p} e^{-\frac{p \alpha|u|^{2}}{2} \omega} \omega(u) d A(u),
$$

for any $z \in \mathbb{C}$ and $f \in H(\mathbb{C})$.

Proof. Let be $p_{0} \in(1, \infty)$ such that $\omega \in A_{p_{0}}^{\text {restricted }}$. By subharmonacity for $\alpha=0$ and by [16, Lemma 1] (see also [5, Lemma 7]) for $\alpha>0$, there is a constant $C>0$ such that

$$
|f(z)|^{\frac{p}{p_{0}}} e^{-\frac{p}{p_{0}} \frac{\alpha|z|^{2}}{2}} \leq C \int_{D(z, t)}|f(u)|^{\frac{p}{p_{0}}} e^{-\frac{p}{p_{0}} \frac{\alpha|u|^{2}}{2}} d A(u),
$$


for any $z \in \mathbb{C}$ and $f \in H(\mathbb{C})$. So,

$$
\begin{aligned}
& |f(z)| \frac{p}{p_{0}} e^{-\frac{p}{p_{0}} \frac{\alpha|z|^{2}}{2}} \\
& \leq C\left(\int_{D(z, t)}|f(u)|^{p} e^{-\frac{p \alpha|u|^{2}}{2}} \omega(u) d A(u)\right)^{1 / p_{0}}\left(\int_{D(z, t)} \omega^{-\frac{p_{0}^{\prime}}{p_{0}}}(u) d A(u)\right)^{1 / p_{0}^{\prime}} \\
& \leq \frac{C}{(\omega(D(z, t)))^{1 / p_{0}}}\left(\int_{D(z, t)}|f(u)|^{p} e^{-\frac{p \alpha|u|^{2}}{2}} \omega(u) d A(u)\right)^{1 / p_{0}} .
\end{aligned}
$$

This finishes the proof.

The next result follows from Lemma 3.1.

Corollary 3.2. Let $0<p, \alpha, t<\infty$ and let be $\omega \in A_{\infty}^{\text {restricted }}$. Then, there exists a constant $C=C(\alpha, p, \omega, t)$ such that

$$
|f(z)| \leq \frac{C e^{\frac{\alpha|z|^{2}}{2}}}{\omega(D(z, t))^{1 / p}}\|f\|_{\mathcal{F}_{\alpha, \omega}^{p}}
$$

for any $z \in \mathbb{C}$ and $f \in H(\mathbb{C})$.

Let us recall that $\mathcal{F}_{\alpha}^{\infty}, \alpha>0$, is the space of the the entire functions $f$ such that

$$
\|f\|_{\mathcal{F}_{\alpha}^{\infty}}=\sup _{z \in \mathbb{C}}|f(z)| e^{-\frac{\alpha}{2}|z|^{2}}<\infty .
$$

Proposition 3.3. Let $\alpha>0$ and $\omega \in A_{\infty}^{\text {restricted }}$. Then:

(i) If $0<p<q \leq \infty$,

$$
\mathcal{F}_{\alpha}^{p} \subset \mathcal{F}_{\alpha}^{q}
$$

(ii) For any $\delta \in(0, \alpha)$,

$$
\mathcal{F}_{\alpha-\delta}^{\infty} \subset \mathcal{F}_{\alpha, \omega}^{p} \subset \mathcal{F}_{\alpha+\delta}^{1} .
$$

Proof. (i) is well known (see for instance [21, Theorem 2.10]). Let us prove (ii). Let $f \in \mathcal{F}_{\alpha-\delta}^{\infty}$. By Lemma 2.8

$$
\|f\|_{\mathcal{F}_{\alpha, \omega}^{p}}^{p} \leq\|f\|_{\mathcal{F}_{\alpha-\delta}^{\infty}}^{p} \int_{\mathbb{C}} e^{-p \delta \frac{|z|^{2}}{2}} \omega(z) d A(z)<\infty
$$

which proves the first embedding.

Now assume that $f \in \mathcal{F}_{\alpha, \omega}^{p}$. Then, by Corollary 3.2 and Lemma 2.9

$$
\|f\|_{\mathcal{F}_{\alpha+\delta}^{1}} \lesssim\|f\|_{\mathcal{F}_{\alpha, \omega}^{p}} \int_{\mathbb{C}} e^{-\delta \frac{|z|^{2}}{2}} \omega(D(z, 1))^{-1 / p} d A(z)<\infty .
$$

This finishes the proof.

The next result is a technical byproduct of Proposition 3.3(i) which will be useful to obtain appropriate estimates for $0<p \leq 1$, in terms of the ones for $p>1$.

Corollary 3.4. Let $g \in \mathcal{F}_{\beta}^{1}, 0<\beta<2 \alpha$. Then for $\theta \in(0,1]$ we have $\left[P_{\alpha}^{+}(|g|)(z)\right]^{\theta} \lesssim P_{\theta \alpha}^{+}\left(|g|^{\theta}\right)(z)$. 
Proof. For $z \in \mathbb{C}$,

$$
\begin{aligned}
{\left[P_{\alpha}^{+}(|g|)(z)\right]^{\theta} } & \asymp\left[\int_{\mathbb{C}}\left|g(w) e^{\alpha w \bar{z}}\right| e^{-\alpha|w|^{2}} d A(w)\right]^{\theta} \\
& \asymp\left\|g(\cdot) e^{\alpha(\cdot) \bar{z}}\right\|_{\mathcal{F}_{2 \alpha}^{1}}^{\theta} \lesssim\left\|g(\cdot) e^{\alpha(\cdot) \bar{z}}\right\|_{\mathcal{F}_{2 \alpha}^{\theta}}^{\theta} \\
& \asymp \int_{\mathbb{C}}|g(w)|^{\theta}\left|e^{\theta \alpha w \bar{z}}\right| e^{-\theta \alpha|w|^{2}} d A(w) \asymp P_{\theta \alpha}^{+}\left(|g|^{\theta}\right)(z) .
\end{aligned}
$$

Note that the condition $\beta<2 \alpha$ ensures that all the terms in the above inequalities are finite.

Lemma 3.5. Assume that $0<\beta<2 \alpha$ and $g \in \mathcal{F}_{\beta}^{1}$. Then, for each $z \in \mathbb{C}$ :

(i) If $\gamma(\alpha, \beta):=\frac{\alpha^{2}}{2 \alpha-\beta}$, then $P_{\alpha}^{+}(|g|)(z) \leq e^{\frac{\gamma(\alpha, \beta)}{2}|z|^{2}}\|g\|_{\mathcal{F}_{\beta}^{1}}$;

(ii) For a non-negative integer $k, g^{(k)}(z)=\alpha^{k} P_{\alpha}\left(\bar{\zeta}^{k} g(\zeta)\right)(z)$;

(iii) If $\gamma>\gamma(\alpha, \beta)$, then $g^{(k)} \in \mathcal{F}_{\gamma}^{1}$.

Proof. Since $0<\beta<2 \alpha$, we have

$$
\mathcal{R}(\alpha \bar{z} \zeta)-\left(\alpha-\frac{\beta}{2}\right)|\zeta|^{2} \leq \alpha|z||\zeta|-\frac{2 \alpha-\beta}{2}|\zeta|^{2} \leq \frac{\alpha^{2}}{2(2 \alpha-\beta)}|z|^{2}
$$

So,

$$
\begin{aligned}
P_{\alpha}^{+}(|g|)(z) & =\int_{\mathbb{C}}|g(\zeta)| e^{-\frac{\beta}{2}|\zeta|^{2}} e^{\mathcal{R}(\alpha \bar{z} \zeta)-\left(\alpha-\frac{\beta}{2}\right)|\zeta|^{2}} d A(\zeta) \\
& \leq e^{\frac{\gamma(\alpha, \beta)}{2}|z|^{2}}\|g\|_{\mathcal{F}_{\beta}^{1}} .
\end{aligned}
$$

Now, fix $z \in \mathbb{C}$ and take $\varepsilon>0$ an a polynomial $\psi$ such that

$$
e^{\frac{\gamma(\alpha, \beta)}{2}|z|^{2}}\|g-\psi\|_{\mathcal{F}_{\beta}^{1}}<\frac{\varepsilon}{2} \quad \text { and } \quad|g(z)-\psi(z)|<\frac{\varepsilon}{2} .
$$

Then, by (3.1)

$$
\begin{aligned}
\left|P_{\alpha}(g)(z)-g(z)\right| & \leq\left|P_{\alpha}(g-\psi)(z)\right|+|\psi(z)-g(z)| \\
& \leq P_{\alpha}^{+}(|g-\psi|)(z)+\frac{\varepsilon}{2} \\
& \leq e^{\frac{\gamma(\alpha, \beta)}{2}|z|^{2}}\|g-\psi\|_{\mathcal{F}_{\beta}^{1}}+\frac{\varepsilon}{2}<\varepsilon,
\end{aligned}
$$

which implies that $g(z)=P_{\alpha}(g)(z)$, for any $z \in \mathbb{C}$. From these results it is easy to check that $g^{(k)}(z)=\alpha^{k} P_{\alpha}\left(\bar{\zeta}^{k} g(\zeta)\right)(z)$.

Finally, observe that $|\zeta|^{k} e^{-\varepsilon|\zeta|^{2}}$ is a bounded function for any $\varepsilon>0$. Thus, $\zeta^{k} g(\zeta) \in \mathcal{F}_{\beta+\varepsilon}^{1}$ for any $\varepsilon>0$. So, (iii) is a consequence of (i) and (ii).

The next lemma provides a representation formula of a holomorphic function in terms of its $k$-th derivatives.

Lemma 3.6. Let $\beta \in(0,2 \alpha), k \in \mathbb{N}$ and $f \in \mathcal{F}_{\beta}^{1}$. Let $T_{m}(f)$ be the Taylor polynomial of degree $m$ for $f$, centered at $z=0$. Then,

$$
f(z)=T_{2 k-1}(f)(z)+R_{2 k}(f)(z),
$$


where

$$
R_{2 k}(f)(z):=\frac{\alpha^{1-k}}{\pi} \int_{\mathbb{C}} \frac{\left(f(w)-T_{2 k-1}(f)(w)\right)^{(k)}}{\bar{w}^{k}} e^{\alpha z \bar{w}} e^{-\alpha|w|^{2}} d A(w) .
$$

In particular, for $k=1$ we have

$$
f(z)=f(0)+f^{\prime}(0) z+\frac{1}{\pi} \int_{\mathbb{C}} \frac{f^{\prime}(w)-f^{\prime}(0)}{\bar{w}} e^{\alpha z \bar{w}} e^{-\alpha|w|^{2}} d A(w) .
$$

Proof. By Lemma 3.5 the function $g_{m}=f-T_{m}(f)$ and its $k$-th derivatives are in $\mathcal{F}_{\beta+\delta}^{1}$ for any $\delta>0$. Since

$$
\left(f(w)-T_{2 k-1}(f)(w)\right)^{(k)} \frac{w^{k}}{|w|^{2 k}}
$$

is bounded on the unit disk, we obtain that this function is in $\mathcal{L}_{\beta+\delta}^{1}$. So, $R_{2 k}(f)$ is a entire function and

$$
\begin{aligned}
& \left(f(w)-T_{2 k-1}(f)(w)\right)^{(k)}=\sum_{j=2 k}^{\infty} \frac{f^{(j)}(0)}{j !} j(j-1) \ldots(j-k+1) w^{j-k} \\
& =w^{k} \sum_{m=0}^{\infty} \frac{f^{(m+2 k)}(0)}{(m+2 k) !}(m+2 k)(m+2 k-1) \ldots(m+k+1) w^{m} .
\end{aligned}
$$

Next, orthogonality gives that

$$
\begin{aligned}
& \frac{\alpha^{1-k}}{\pi} \int_{\mathbb{C}} \frac{w^{k+m} w^{k}}{|w|^{2 k}} e^{\alpha z \bar{w}} e^{-\alpha|w|^{2}} d A(w) \\
& =\frac{\alpha^{1-k}}{\pi} \int_{\mathbb{C}}|w|^{2 k+2 m} \frac{(\alpha z)^{2 k+m}}{(2 k+m) !} e^{-\alpha|w|^{2}} d A(w) \\
& =\frac{2 \alpha^{1+k+m}}{(2 k+m) !}\left(\int_{0}^{\infty} r^{2 k+2 m+1} e^{-\alpha r^{2}} d r\right) z^{2 k+m} \\
& =\frac{\alpha^{k+m}}{(2 k+m) !}\left(\int_{0}^{\infty} \frac{t^{k+m}}{\alpha^{k+m}} e^{-t} d t\right) z^{2 k+m}=\frac{(k+m) !}{(2 k+m) !} z^{2 k+m}
\end{aligned}
$$

where in the previous to the last equality we have made the change of variables $t=\alpha r^{2}$. Bearing in mind this calculation, the fact that $(f(w)-$ $\left.T_{2 k-1}(f)(w)\right)^{(k)} \frac{w^{k}}{|w|^{2 k}}$ is bounded on $\mathbb{D}$, we deduce that

$$
\begin{aligned}
& \frac{\alpha^{1-k}}{\pi} \int_{\mathbb{C}} \frac{\left(f(w)-T_{2 k-1}(f)(w)\right)^{(k)}}{\bar{w}^{k}} e^{\alpha z \bar{w}} e^{-\alpha|w|^{2}} d A(w) \\
& =\sum_{m=0}^{\infty}(m+2 k) \ldots(m+k+1) \frac{f^{(m+2 k)}(0)}{(m+2 k) !} \frac{\alpha^{1-k}}{\pi} \int_{\mathbb{C}} \frac{w^{k+m} w^{k}}{|w|^{2 k}} e^{\alpha z \bar{w}} e^{-\alpha|w|^{2}} d A(w) \\
& =\sum_{m=0}^{\infty} \frac{f^{(m+2 k)}(0)}{(m+2 k) !} z^{m+2 k}=f(z)-T_{2 k-1}(f)(z),
\end{aligned}
$$

which gives the desired result. 
Remark 3.7. By Proposition 3.3, if $\omega \in A_{\infty}^{\text {restricted }}$ then $\mathcal{F}_{\alpha, \omega}^{p} \subset \mathcal{F}_{\alpha+\delta}^{1}$ for any $\delta>0$. So, all the theses in Corollary 3.4, Lemma 3.5 and Lemma 3.6 remain true replacing $\mathcal{F}_{\beta}^{1}$ by $\mathcal{F}_{\alpha, \omega}^{p}$ in the corresponding hypotheses.

3.2. Main results. First, we will restrict ourselves to the case of the first derivative.

Lemma 3.8. Let $p>0$ and $0<\beta<2 \alpha$. If $\omega \in A_{\infty}^{\text {restricted }}$ and $g \in \mathcal{F}_{\beta, \omega}^{p}$, then $\left\|P_{\alpha}^{+}(|g|)\right\|_{\mathcal{L}_{\alpha, \omega}^{p}} \lesssim\|g \mid\|_{\mathcal{F}_{\alpha, \omega}^{p}}$.

Proof. By Proposition 2.2(iii), we can assume that $\omega \in A_{p_{0}}^{\text {restricted }}$ for some $p_{0}>\max \{p, 1\}$. Remark 3.7, Corollary 3.4 with $\theta=p / p_{0}$ together Theorem A give

$$
\begin{aligned}
\left\|P_{\alpha}^{+}(|g|)\right\|_{\mathcal{L}_{\alpha, \omega}^{p}} & \lesssim\left\|\left(P_{p \alpha / p_{0}}^{+}\left(|g|^{p / p_{0}}\right)\right)^{p_{0} / p}\right\|_{\mathcal{L}_{\alpha, \omega}^{p}} \asymp\left\|P_{p \alpha / p_{0}}^{+}\left(|g|^{p / p_{0}}\right)\right\|_{\mathcal{L}_{\alpha p / p_{0}, \omega}^{p_{0}}} \\
& \lesssim\left\||g|^{p / p_{0}}\right\|_{\mathcal{L}_{\alpha p / p_{0}, \omega}^{p_{0}}} \asymp\|g\|_{\mathcal{F}_{\alpha, \omega}^{p}} .
\end{aligned}
$$

This ends the proof.

Proposition 3.9. Let $0<p<\infty, \alpha>0$ and $\omega \in A_{\infty}^{\text {restricted }}$. Then,

$$
|f(0)|+\left\|f^{\prime}\right\|_{\mathcal{F}_{\alpha, \omega_{p}}^{p}} \lesssim\|f\|_{\mathcal{F}_{\alpha, \omega}^{p},}, \quad f \in H(\mathbb{C}) .
$$

Proof. By Lemma 3.1, there is $C=C(p, \omega, \alpha)$ such that

$$
|f(0)|^{p} \leq C \int_{|z| \leq 1}|f(z)|^{p} e^{-p \frac{\alpha}{2}|z|^{2}} \omega(z) d A(z) \lesssim\|f\|_{\mathcal{F}_{\alpha, \omega}^{p}}^{p} .
$$

So, to conclude the proof we need to show that

$$
\left\|f^{\prime}\right\|_{\mathcal{F}_{\alpha, \omega_{p}}^{p}} \lesssim\|f\|_{\mathcal{F}_{\alpha, \omega}^{p}} .
$$

By Lemma 2.1, $\omega_{p}(z)=\frac{\omega(z)}{(1+|z|)^{p}} \in A_{\infty}^{\text {restricted }}$. So, by Remark 3.7, Lemma 3.5 and Lemma 3.8

$$
\left\|f^{\prime}\right\|_{\mathcal{F}_{\alpha, \omega_{p}}^{p}} \lesssim\left\|P_{\alpha}^{+}(|w f(w)|)\right\|_{\mathcal{L}_{\alpha, \omega_{p}}^{p}} \lesssim\|w f(w)\|_{\mathcal{F}_{\alpha, \omega_{p}}^{p}} \lesssim\|f\|_{\mathcal{F}_{\alpha, \omega}^{p}} .
$$

This ends the proof.

Proposition 3.10. Let $0<p<\infty, \alpha>0$ and $\omega \in A_{\infty}^{\text {restricted }}$. Then,

$$
\|f\|_{\mathcal{F}_{\alpha, \omega}^{p}} \lesssim|f(0)|+\left\|f^{\prime}\right\|_{\mathcal{F}_{\alpha, \omega_{p}}^{p}}, \quad f \in H(\mathbb{C}) .
$$

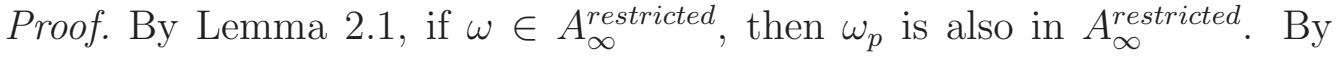
Lemma 2.1 and Proposition 3.3, $f^{\prime} \in \mathcal{F}_{\alpha, \omega_{p}}^{p} \subset \mathcal{F}_{\alpha+\delta}^{1}$, for any $0<\delta<\alpha$. So, by (1.1), we have $f \in \mathcal{F}_{\alpha+\delta}^{1}$. Thus, the representation formula in Lemma 3.6 holds and Lemma 2.8 yield

$$
\|f\|_{\mathcal{F}_{\alpha, \omega}^{p}} \lesssim|f(0)|+\left\|f^{\prime}(0) z\right\|_{\mathcal{F}_{\alpha, \omega}^{p}}+\left\|R_{2}(f)\right\|_{\mathcal{F}_{\alpha, \omega}^{p}},
$$

where

$$
R_{2}(f)(z)=\frac{1}{\pi} \int_{\mathbb{C}} \frac{f^{\prime}(w)-f^{\prime}(0)}{\bar{w}} e^{\alpha z \bar{w}} e^{-\alpha|w|^{2}} d A(w)
$$


By Lemma 3.1 and Proposition 3.3,

$$
\left|f^{\prime}(0)\right| \lesssim\left\|f^{\prime}\right\|_{\mathcal{F}_{\alpha+\delta}^{1}} \lesssim\left\|f^{\prime}\right\|_{\mathcal{F}_{\alpha, \omega_{p}}}, \quad \delta>0
$$

Since $\|z\|_{\mathcal{F}_{\alpha, \omega}^{p}}<\infty$, we obtain

$$
|f(0)|+\left\|f^{\prime}(0) z\right\|_{\mathcal{F}_{\alpha, \omega}^{p}} \lesssim|f(0)|+\left\|f^{\prime}\right\|_{\mathcal{F}_{\alpha, \omega_{p}}} .
$$

Now, let us deal with the last term above. Let $g(\zeta):=\frac{f^{\prime}(\zeta)-f^{\prime}(0)}{\zeta}$. Let us observe that if $|\zeta| \leq 1$ and $\delta \in(0, \alpha)$, it follows from Proposition 3.3 and Lemma 2.1

$$
\begin{aligned}
|g(\zeta)| & =\left|\int_{0}^{1} f^{\prime \prime}(t \zeta) d t\right| \leq \sup _{|w| \leq 1}\left|f^{\prime \prime}(w)\right| \\
& \lesssim \int_{D(0,2)}\left|f^{\prime}(w)\right| e^{-(\alpha+\delta)|w|^{2}} d A(w) \lesssim\left\|f^{\prime}\right\|_{\mathcal{F}_{\alpha+\delta}^{1}} \lesssim\left\|f^{\prime}\right\|_{\mathcal{F}_{\alpha, \omega_{p}}^{p}},
\end{aligned}
$$

where in the second to last inequality we use that $e^{-(\alpha+\delta)|w|^{2}} \asymp 1$ on $D(0,2)$.

Moreover, if $|\zeta|>1$,

$$
|g(\zeta)| \leq 2 \frac{\left|f^{\prime}(\zeta)\right|+\left|f^{\prime}(0)\right|}{1+|\zeta|} \lesssim \frac{\left|f^{\prime}(\zeta)\right|}{1+|\zeta|}+\left|f^{\prime}(0)\right| .
$$

Thus, $g$ is a entire function which satisfies

$$
|g(\zeta)| \lesssim \frac{\left|f^{\prime}(\zeta)\right|}{1+|\zeta|}+\left\|f^{\prime}\right\|_{\mathcal{F}_{\alpha, \omega_{p}}^{p}}
$$

which shows that $g \in \mathcal{F}_{\alpha, \omega}^{p}$ and

$$
\|g\|_{\mathcal{F}_{\alpha, \omega}^{p}} \lesssim\left\|f^{\prime}\right\|_{\mathcal{F}_{\alpha, \omega_{p}}^{p}} .
$$

Therefore, by Lemma 3.8

$$
\left\|R_{2}(f)\right\|_{\mathcal{F}_{\alpha, \omega}^{p}} \lesssim\left\|P_{\alpha}^{+}(|g|)(z)\right\|_{\mathcal{L}_{\alpha, \omega}^{p}} \lesssim\|g\|_{\mathcal{F}_{\alpha, \omega}^{p}} \lesssim\left\|f^{\prime}\right\|_{\mathcal{F}_{\alpha, \omega_{p}}^{p}} .
$$

This ends the proof.

Proof of Theorem 1.1. It follows from Propositions 3.9 and 3.10 that

$$
\|f\|_{\mathcal{F}_{\alpha, \omega}^{p}} \asymp|f(0)|+\left\|f^{\prime}\right\|_{\mathcal{F}_{\alpha, \omega_{p}}^{p}} .
$$

By Lemma 2.1, $\omega_{k p}=\left(\omega_{(k-1) p}\right)_{p} \in A_{\infty}^{\text {restricted }}$ for any $k \in \mathbb{N}$. Therefore, consecutive iterations of (3.3) give that

$$
\|f\|_{\mathcal{F}_{\alpha, \omega}^{p}} \asymp \sum_{j=1}^{k-1}\left|f^{(j)}(0)\right|+\left\|f^{(k)}\right\|_{\mathcal{F}_{\alpha, \omega_{k p}}^{p}} .
$$

This finishes the proof.

\section{Carleson measures on Weighted Fock-Sobolev spaces}

We begin proving appropriate norm estimates for the family of test functions employed in the proof of Theorem 1.2. 
4.1. Test functions. We will write $K_{\alpha, a}(z)=K_{a}(z)=e^{\alpha \bar{a} z}$ for the reproducing kernels of the classical Fock space $\mathcal{F}_{\alpha}^{2}$.

Proposition 4.1. Let $\alpha, p \in(0, \infty)$ and $\omega \in A_{\infty}^{\text {restricted }}$. Then,

$$
\left\|K_{a}\right\|_{\mathcal{F}_{\alpha, \omega}^{p}}^{p} \asymp e^{\alpha \frac{p|a|^{2}}{2}} \omega(D(a, 1)), \quad a \in \mathbb{C} .
$$

Proof. Bearing in mind that

$$
\left\|K_{a}\right\|_{\mathcal{F}_{\alpha, \omega}^{p}}^{p}=e^{\alpha \frac{p|a|^{2}}{2}} \int_{\mathbb{C}} e^{\alpha \frac{-p|z-a|^{2}}{2}} \omega(z) d A(z)=e^{\alpha \frac{p|a|^{2}}{2}} \int_{\mathbb{C}} e^{\alpha \frac{-p|u|^{2}}{2}} \omega_{[a]}(u) d A(u),
$$

the proof follows from Lemma 2.4 and Lemma 2.8.

Proposition 4.2. Let $\alpha, p \in(0, \infty)$ and $\omega \in A_{\infty}^{\text {restricted }}$. Then, for any sequence $\left\{a_{\nu}\right\}_{\nu \in r \mathbb{Z}^{2}} \in l^{p}$,

$$
f(z)=\sum_{\nu \in r \mathbb{Z}^{2}} a_{\nu} \frac{K_{\nu}(z)}{\left\|K_{\nu}\right\|_{\mathcal{F}_{\alpha, \omega}^{p}}^{p}} \in \mathcal{F}_{\alpha, \omega}^{p}
$$

with $\|f\|_{\mathcal{F}_{\alpha, \omega}^{p}} \lesssim\left\|\left\{a_{\nu}\right\}\right\|_{l^{p}}$.

Proof. If $0<p \leq 1$, then

$$
\|f\|_{\mathcal{F}_{\alpha, \omega}^{p}}^{p} \leq \int_{\mathbb{C}} \sum_{\nu \in r \mathbb{Z}^{2}} \frac{\left|a_{\nu}\right|^{p}\left|K_{\nu}(z)\right|^{p}}{\left\|K_{\nu}\right\|_{\mathcal{F}_{\alpha, \omega}^{p}}^{p}} e^{\alpha \frac{-p|z|^{2}}{2}} \omega(z) d A(z)=\sum_{\nu \in r \mathbb{Z}^{2}}\left|a_{\nu}\right|^{p} .
$$

If $1<p<\infty$, then bearing in mind Proposition 4.1,

$$
\begin{aligned}
& \|f\|_{\mathcal{F}_{\alpha, \omega}^{p}}^{p} \\
& \lesssim \int_{\mathbb{C}}\left(\sum_{\nu \in r \mathbb{Z}^{2}} \frac{\left|a_{\nu}\right|\left|K_{\nu}(z)\right|}{e^{\alpha \frac{|\nu|^{2}}{2}} \omega(D(\nu, 1))^{1 / p}}\right)^{p} e^{\alpha \frac{-p|z|^{2}}{2}} \omega(z) d A(z) \\
& \lesssim \int_{\mathbb{C}}\left(\sum_{\nu \in r \mathbb{Z}^{2}} \frac{\left|a_{\nu}\right|^{p}\left|K_{\nu}(z)\right|}{e^{\alpha \frac{|\nu|^{2}}{2}} \omega(D(\nu, 1))}\right)\left(\sum_{\nu \in r \mathbb{Z}^{2}} \frac{\left|K_{\nu}(z)\right|}{e^{\alpha \frac{|\nu|^{2}}{2}}}\right)^{p-1} e^{\alpha \frac{-p|z|^{2}}{2}} \omega(z) d A(z) .
\end{aligned}
$$

Next, by Lemma 3.1

$$
\begin{aligned}
\sum_{\nu \in r \mathbb{Z}^{2}}\left|K_{\nu}(z)\right| e^{-\alpha \frac{\left.\nu\right|^{2}}{2}} & \lesssim \sum_{\nu \in r \mathbb{Z}^{2}} \int_{D\left(\nu, \frac{r}{2}\right)}\left|K_{z}(u)\right| e^{-\alpha \frac{|u|^{2}}{2}} d A(u) \\
& \lesssim \int_{\mathbb{C}}\left|K_{z}(u)\right| e^{-\alpha \frac{|u|^{2}}{2}} d A(u) \asymp e^{\alpha \frac{|z|^{2}}{2}}, \quad z \in \mathbb{C} .
\end{aligned}
$$

So, by Proposition 4.1

$$
\begin{aligned}
\|f\|_{\mathcal{F}_{\alpha, \omega}^{p}}^{p} & \lesssim \sum_{\nu \in r \mathbb{Z}^{2}} \frac{\left|a_{\nu}\right|^{p}}{e^{\alpha \frac{|\nu|^{2}}{2}} \omega(D(\nu, 1))} \int_{\mathbb{C}}\left|K_{\nu}(z)\right| e^{-\alpha \frac{|z|^{2}}{2}} \omega(z) d A(z) \\
& \asymp \sum_{\nu \in r \mathbb{Z}^{2}}\left|a_{\nu}\right|^{p} .
\end{aligned}
$$

This finishes the proof. 
4.2. Proof of Theorem 1.2. First, we will deal with the case $n=0$. Later on, we will use Theorem 1.1 to deduce the rest of cases from this particular one.

\subsection{Case $p \leq q$.}

Theorem 4.3. Let $\alpha \in(0, \infty), \omega \in A_{\infty}^{\text {restricted }}$ and let $\mu$ be a positive Borel measure on $\mathbb{C}$. For $0<p \leq q<\infty$, the following conditions are equivalent;

(i) $\mu$ is a q-Carleson measure for $\mathcal{F}_{\alpha, \omega}^{p}$;

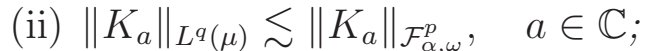

(iii) $\int_{D(a, 1)} e^{\alpha \frac{q}{2}|z|^{2}} d \mu(z) \lesssim(\omega(D(a, 1)))^{\frac{q}{p}}, \quad a \in \mathbb{C}$.

Moreover,

$$
\left\|I_{d}\right\|_{\mathcal{F}_{\alpha, \omega}^{p} \rightarrow L^{q}(\mu)}^{q} \asymp \sup _{a \in \mathbb{C}} \frac{\left\|K_{a}\right\|_{L^{q}(\mu)}^{q}}{\left\|K_{a}\right\|_{L^{p}(\mu)}^{q}} \asymp \sup _{a \in \mathbb{C}} \frac{\int_{D(a, 1)} e^{\alpha \frac{q}{2}|z|^{2}} d \mu(z)}{(\omega(D(a, 1)))^{\frac{q}{p}}} .
$$

Proof. Let us denote $G:=\sup _{a \in \mathbb{C}} \frac{\int_{D(a, 1)} e^{\alpha \frac{q}{2}|z|^{2}} d \mu(z)}{\left(\int_{D(a, 1)} \omega(z) d A(z)\right)^{\frac{q}{p}}}$.

(i) $\Rightarrow$ (ii) and the inequality $\left\|I_{d}\right\|_{\mathcal{F}_{\alpha, \omega}^{p} \rightarrow L^{q}(\mu)}^{q} \geq \sup _{a \in \mathbb{C}} \frac{\left\|K_{a}\right\|_{L^{q}(\mu)}^{q}}{\left\|K_{a}\right\|_{L^{p}(\mu)}^{q}}$ are clear.

Assume (ii). Then, by Proposition 4.1, for any $a \in \mathbb{C}$,

$$
\int_{\mathbb{C}} e^{q \alpha \mathcal{R}(\bar{a} z)} e^{-\alpha \frac{q}{2}|a|^{2}} d \mu(z)=\int_{\mathbb{C}} e^{\alpha \frac{q}{2}|z|^{2}} e^{-\alpha \frac{q}{2}|z-a|^{2}} d \mu(z) \lesssim(\omega(D(a, 1)))^{\frac{q}{p}} .
$$

So, for any $a \in \mathbb{C}$,

$$
(\omega(D(a, 1)))^{\frac{q}{p}} \gtrsim \int_{D(a, 1)} e^{\alpha \frac{q}{2}|z|^{2}} e^{-\alpha \frac{q}{2}|z-a|^{2}} d \mu(z) \asymp \int_{D(a, 1)} e^{\alpha \frac{q}{2}|z|^{2}} d \mu(z),
$$

which gives (iii) and the inequality

$$
\sup _{a \in \mathbb{C}} \frac{\left\|K_{a}\right\|_{L^{q}(\mu)}^{q}}{\left\|K_{a}\right\|_{L^{p}(\mu)}^{q}} \gtrsim \sup _{a \in \mathbb{C}} \frac{\int_{D(a, 1)} e^{\alpha \frac{q}{2}|z|^{2}} d \mu(z)}{(\omega(D(a, 1)))^{\frac{q}{p}}} .
$$

Now, let us prove (iii) $\Rightarrow$ (i). It follows from (iii) and Lemma 3.1 that

$$
\begin{array}{rl}
\int_{\mathbb{C}}|f(z)|^{q} & d \mu(z) \leq \sum_{\nu \in \mathbb{Z}^{2}} \int_{D(\nu, 1)}|f(z)|^{q} d \mu(z) \\
& =\sum_{\nu \in \mathbb{Z}^{2}} \int_{D(\nu, 1)}\left|f(z) e^{-\alpha \frac{|z|^{2}}{2}}\right|^{q} e^{q \alpha \frac{|z|^{2}}{2}} d \mu(z) \\
& \lesssim \sum_{\nu \in \mathbb{Z}^{2}} \int_{D(\nu, 1)}\left(\frac{\int_{D(z, 1)}|f(u)|^{p} e^{-p \alpha \frac{|u|^{2}}{2}} \omega(u) d A(u)}{\omega(D(z, 1))}\right)^{\frac{q}{p}} e^{q \alpha \frac{|z|^{2}}{2}} d \mu(z) .
\end{array}
$$


By Lemma B (see also Remark 2.3), Lemma 2.8 and the fact that $D(z, 1) \subset$ $D(\nu, 2)$ for $z \in D(\nu, 1)$, we have

$$
\begin{aligned}
& \int_{\mathbb{C}}|f(z)|^{q} d \mu(z) \\
& \lesssim \sum_{\nu \in \mathbb{Z}^{2}}\left(\frac{\int_{D(\nu, 2)}|f(u)|^{p} e^{-p \alpha \frac{|u|^{2}}{2}} \omega(u) d A(u)}{\omega(D(\nu, 1))}\right)^{\frac{q}{p}} \int_{D(\nu, 1)} e^{q \alpha \frac{|z|^{2}}{2}} d \mu(z) \\
& \lesssim G \sum_{\nu \in \mathbb{Z}^{2}}\left(\int_{D(\nu, 2)}|f(u)|^{p} e^{-p \alpha \frac{|u|^{2}}{2}} \omega(u) d A(u)\right)^{\frac{q}{p}} .
\end{aligned}
$$

Finally, using Minkowski's inequality and the fact that $\{D(\nu, 2)\}_{\nu \in \mathbb{Z}^{2}}$ is a covering of $\mathbb{C}$ which overlaps finitely many times, it follows that

$$
\|f\|_{L^{q}(\mu)}^{q} \lesssim G\|f\|_{\mathcal{F}_{\alpha, \omega}^{p}}^{q}, \quad f \in H(\mathbb{C}),
$$

which implies that $\left\|I_{d}\right\|_{\mathcal{F}_{\alpha, \omega}^{p} \rightarrow L^{q}(\mu)}^{q} \lesssim G$ and finishes the proof.

\subsection{Case $q<p$.}

Theorem 4.4. Let be $\alpha \in(0, \infty), \omega \in A_{\infty}^{\text {restricted }}$ and $\mu$ a positive Borel measure on $\mathbb{C}$. For $0<q<p<\infty$, the following conditions are equivalent;

(i) $\mu$ is a q-Carleson measure for $\mathcal{F}_{\alpha, \omega}^{p}$;

(ii) The function

$$
H(u)=\frac{\int_{D(u, 1)} e^{\frac{q \alpha|z|^{2}}{2}} d \mu(z)}{\omega(D(u, 1))} \in L^{\frac{p}{p-q}}(\mathbb{C}, \omega) .
$$

Moreover,

$$
\left\|I_{d}\right\|_{\mathcal{F}_{\alpha, \omega}^{p} \rightarrow L^{q}(\mu)}^{q} \asymp\|H\|_{L^{p /(p-q)}(\mathbb{C}, \omega)} .
$$

Proof. This proof uses ideas from [13, Theorem 1]. Assume that (ii) holds. Then, by Lemmas 3.1 and B (see also Remark 2.3), the equivalence (2.5) and Hölder's inequality, we obtain

$$
\begin{aligned}
\int_{\mathbb{C}}|f(z)|^{q} d \mu(z) & \lesssim \int_{\mathbb{C}}\left[\frac{\int_{D(z, 1)}|f(u)|^{q} e^{\frac{-q \alpha|u|^{2}}{2} \omega(u) d A(u)}}{\omega(D(z, 2))}\right] e^{\frac{q \alpha|z|^{2}}{2}} d \mu(z) \\
& \lesssim \int_{\mathbb{C}}|f(u)|^{q} e^{\frac{-q \alpha|u|^{2}}{2}}\left[\frac{\int_{D(u, 1)} e^{\frac{q \alpha|z|^{2}}{2}} d \mu(z)}{\omega(D(u, 1))}\right] \omega(u) d A(u) \\
& \leq\|f\|_{\mathcal{F}_{\alpha, \omega}^{p}}^{q} \int_{\mathbb{C}}\left[\frac{\int_{D(u, 1)} e^{\frac{q \alpha|z|^{2}}{2}} d \mu(z)}{\omega(D(u, 1))}\right]^{\frac{p}{p-q}} \omega(u) d A(u),
\end{aligned}
$$


which gives (i) and the inequality

$$
\left\|I_{d}\right\|_{\mathcal{F}_{\alpha, \omega}^{p} \rightarrow L^{q}(\mu)}^{q} \lesssim \int_{\mathbb{C}}\left[\frac{\int_{D(u, 1)} e^{\frac{q \alpha|z|^{2}}{2}} d \mu(z)}{\omega(D(u, 1))}\right]^{\frac{p}{p-q}} \omega(u) d A(u) .
$$

Reciprocally, assume that (i) holds and let us consider the functions

$$
G_{t}(z)=\sum_{\nu \in \mathbb{Z}^{2}} a_{\nu} R_{\nu}(t) \frac{K_{\nu}(z)}{\left\|K_{\nu}\right\|_{\mathcal{F}_{\alpha, \omega}^{p}}}
$$

where $R_{\nu}(t)$ is a sequence of Rademacher functions (see page 336 of [13], or Appendix A of [8]). So, using $G_{t}$ as test functions, applying Khinchine's inequality and using Proposition 4.2, we deduce that

$$
\begin{aligned}
\int_{\mathbb{C}}\left(\sum_{\nu \in \mathbb{Z}^{2}}\left|a_{\nu}\right|^{2} \frac{\left|K_{\nu}(z)\right|^{2}}{\left\|K_{\nu}\right\|_{\mathcal{F}_{\alpha, \omega}^{p}}^{2}}\right)^{\frac{q}{2}} d \mu(z) & \asymp \int_{\mathbb{C}} \int_{0}^{1}\left|G_{t}(z)\right|^{q} d t d \mu(z) \\
& \lesssim\left\|I_{d}\right\|_{\mathcal{F}_{\alpha, \omega}^{p} \rightarrow L^{q}(\mu)}^{q}\left\|a_{\nu}\right\|_{l^{p}}^{q}
\end{aligned}
$$

Next, bearing in mind that $\{D(\nu, 2)\}_{\nu \in \mathbb{Z}^{2}}$ is a covering of $\mathbb{C}$ which overlaps finitely many times, and using Proposition 4.1 it follows that

$$
\begin{aligned}
\int_{\mathbb{C}} & \left(\sum_{\nu \in r \mathbb{Z}^{2}}\left|a_{\nu}\right|^{2} \frac{\left|K_{\nu}(z)\right|^{2}}{\left\|K_{\nu}\right\|_{\mathcal{F}_{\alpha, \omega}^{p}}^{2}}\right)^{\frac{q}{2}} d \mu(z) \\
& \geq \int_{\mathbb{C}}\left(\sum_{\nu \in r \mathbb{Z}^{2}}\left|a_{\nu}\right|^{2} \frac{\left|K_{\nu}(z)\right|^{2}}{\left\|K_{\nu}\right\|_{\mathcal{F}_{\alpha, \omega}^{p}}^{2}} \chi_{D(\nu, 2)}(z)\right)^{\frac{q}{2}} d \mu(z) \\
& \gtrsim \int_{\mathbb{C}} \sum_{\nu \in \mathbb{Z}^{2}}\left|a_{\nu}\right|^{q} \frac{\left|K_{\nu}(z)\right|^{q}}{\left\|K_{\nu}\right\|_{\mathcal{F}_{\alpha, \omega}^{p}}^{p}} \chi_{D(\nu, 2)}(z) d \mu(z) \\
& =\sum_{\nu \in \mathbb{Z}^{2}} \frac{\left|a_{\nu}\right|^{q}}{\left\|K_{\nu}\right\|_{\mathcal{F}_{\alpha, \omega}^{p}}^{q}} \int_{D(\nu, 2)}\left|K_{\nu}(z)\right|^{q} d \mu(z) \\
& \asymp \sum_{\nu \in \mathbb{Z}^{2}} \frac{\left|a_{\nu}\right|^{q} e^{-\frac{q \alpha|\nu|^{2}}{2}}}{(\omega(D(\nu, 1)))^{\frac{q}{p}}} \int_{D(\nu, 2)}\left|K_{\nu}(z)\right|^{q} d \mu(z) \\
& \asymp \sum_{\nu \in \mathbb{Z}^{2}} \frac{\left|a_{\nu}\right|^{q}}{(\omega(D(\nu, 1)))^{\frac{q}{p}}} \int_{D(\nu, 2)} e^{\frac{q \alpha|z|^{2}}{2}} d \mu(z) .
\end{aligned}
$$

These estimates together with (4.1) and the classical duality relation $\left(l^{\frac{p}{q}}\right)^{\star} \simeq l^{\left(\frac{p}{q}\right)^{\prime}}=l^{\frac{p}{p-q}}, p>q$, gives that

$$
\begin{aligned}
\sum_{\nu \in \mathbb{Z}^{2}}\left(\frac{\int_{D(\nu, 2)} e^{\frac{q \alpha|z|^{2}}{2}} d \mu(z)}{\omega(D(\nu, 1))}\right)^{\frac{p}{p-q}} \omega(D(\nu, 1)) & =\sum_{\nu \in \mathbb{Z}^{2}}\left(\frac{\int_{D(\nu, 2)} e^{\frac{q \alpha|z|^{2}}{2}} d \mu(z)}{(\omega(D(\nu, 1)))^{\frac{q}{p}}}\right)^{\frac{p}{p-q}} \\
& \lesssim\left\|I_{d}\right\|_{\mathcal{F}_{\alpha, \omega}^{p} \rightarrow L^{q}(\mu)}^{q} .
\end{aligned}
$$


So, bearing in mind Remark 2.3

$$
\begin{aligned}
& \int_{\mathbb{C}}\left[\frac{\int_{D(u, 1)} e^{\frac{q \alpha|z|^{2}}{2}} d \mu(z)}{\omega(D(u, 1))}\right]^{\frac{p}{p-q}} \omega(u) d A(u) \\
& \lesssim \sum_{\nu \in \mathbb{Z}^{2}}\left[\frac{\int_{D(\nu, 2)} e^{\frac{q \alpha|z|^{2}}{2}} d \mu(z)}{\omega(D(\nu, 1))}\right]^{\frac{p}{p-q}} \omega(D(\nu, 1)) \lesssim\left\|I_{d}\right\|_{\mathcal{F}_{\alpha, \omega}^{p} \rightarrow L^{q}(\mu)}^{q} .
\end{aligned}
$$

This finishes the proof.

Proof of Theorem 1.2.

The case $n=0$ follows from Theorem 4.3 and Theorem 4.4. Next, by Lemma 2.1

$$
\omega \in A_{\infty}^{\text {restricted }} \Leftrightarrow \omega_{\gamma}(z)=\frac{\omega(z)}{(1+|z|)^{\gamma}} \in A_{\infty}^{\text {restricted }}
$$

So the equivalece (ii) $\Leftrightarrow$ (iii) for $p \leq q$ follows from Theorem 4.3 and the same equivalence for $q<p$ follows from Theorem 4.4.

Now let us prove $(\mathrm{i}) \Leftrightarrow(\mathrm{ii})$. First, assume that $n \in \mathbb{N}$ and $I_{d}: \mathcal{F}_{\alpha, \omega_{n p}}^{p} \rightarrow$ $L^{q}(\mu)$ is bounded, that is

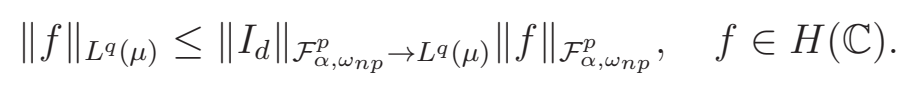

Then, applying the above inequality to $f^{(n)}$, for each $f \in H(\mathbb{C})$, and using Theorem 1.1 we deduce that

$$
\begin{aligned}
\left\|f^{(n)}\right\|_{L^{q}(\mu)} & \leq\left\|I_{d}\right\|_{\mathcal{F}_{\alpha, \omega_{n p}}^{p} \rightarrow L^{q}(\mu)}\left\|f^{(n)}\right\|_{\mathcal{F}_{\alpha, \omega_{n} p}^{p}} \\
& \leq\left\|I_{d}\right\|_{\mathcal{F}_{\alpha, \omega_{n p}}^{p} \rightarrow L^{q}(\mu)}\left(\sum_{k=0}^{n-1}\left|f^{(k)}(0)\right|^{p}+\left\|f^{(n)}\right\|_{\mathcal{F}_{\alpha, \omega_{n p}}^{p}}^{p}\right)^{1 / p} \\
& \asymp\left\|I_{d}\right\|_{\mathcal{F}_{\alpha, \omega_{n p}}^{p} \rightarrow L^{q}(\mu)}\|f\|_{\mathcal{F}_{\alpha, \omega}^{p}},
\end{aligned}
$$

that is $D^{(n)}: \mathcal{F}_{\alpha, \omega}^{p} \rightarrow L^{q}(\mu)$ is bounded and $\left\|D^{(n)}\right\|_{\mathcal{F}_{\alpha, \omega}^{p} \rightarrow L^{q}(\mu)} \lesssim\left\|I_{d}\right\|_{\mathcal{F}_{\alpha, \omega_{n p}}^{p} \rightarrow L^{q}(\mu)}$.

Reciprocally, assume that $D^{(n)}: \mathcal{F}_{\alpha, \omega}^{p} \rightarrow L^{q}(\mu)$ is bounded, that is,

$$
\left\|f^{(n)}\right\|_{L^{q}(\mu)} \leq\left\|D^{(n)}\right\|_{\mathcal{F}_{\alpha, \omega}^{p} \rightarrow L^{q}(\mu)}\|f\|_{\mathcal{F}_{\alpha, \omega}^{p}}, \quad f \in H(\mathbb{C}) .
$$

Then, by Theorem 1.1,

$$
\left\|f^{(n)}\right\|_{L^{q}(\mu)} \leq\left\|D^{(n)}\right\|_{\mathcal{F}_{\alpha, \omega}^{p} \rightarrow L^{q}(\mu)}\left(\sum_{k=0}^{n-1}\left|f^{(k)}(0)\right|^{p}+\left\|f^{(n)}\right\|_{\mathcal{F}_{\alpha, \omega_{n p}}^{p}}^{p}\right)^{1 / p}, \quad f \in H(\mathbb{C}) .
$$

Now, replacing in the above inequality $f$ by $D^{(-n)}(f)$, it follows that

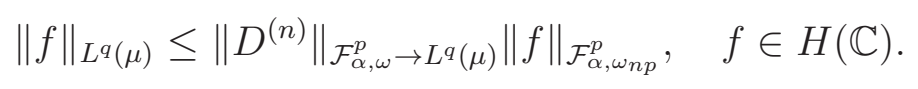

Therefore, $I_{d}: \mathcal{F}_{\alpha, \omega_{n p}}^{p} \rightarrow L^{q}(\mu)$ is bounded and

$$
\left\|I_{d}\right\|_{\mathcal{F}_{\alpha, \omega_{n p}}^{p} \rightarrow L^{q}(\mu)}\|\lesssim\| D^{(n)} \|_{\mathcal{F}_{\alpha, \omega}^{p} \rightarrow L^{q}(\mu)} .
$$


Consequently, we have already proved (i) $\Leftrightarrow($ ii $) \Leftrightarrow($ iii), as well as (1.2) and (1.4), for any $n \in \mathbb{N} \cup\{0\}$.

Second, assume that $n$ is a negative integer and $D^{(n)}: \mathcal{F}_{\alpha, \omega}^{p} \rightarrow L^{q}(\mu)$ is bounded, that is,

$$
\left\|D^{(n)}(f)\right\|_{L^{q}(\mu)} \leq\left\|D^{(n)}\right\|_{\mathcal{F}_{\alpha, \omega}^{p} \rightarrow L^{q}(\mu)}\|f\|_{\mathcal{F}_{\alpha, \omega}^{p}}, \quad f \in H(\mathbb{C}) .
$$

Then, applying the above inequality to $f^{(-n)}$ and using Theorem 1.1 (with $\left.\omega_{n p}\right)$ it follows that

$$
\begin{aligned}
\|f\|_{L^{q}(\mu)} & \lesssim\left(\left\|D^{(n)}\right\|_{\mathcal{F}_{\alpha, \omega}^{p} \rightarrow L^{q}(\mu)}+C_{\mu, n}\right)\left(\sum_{k=0}^{-n-1}\left|f^{(k)}(0)\right|^{p}+\left\|f^{(-n)}\right\|_{\mathcal{F}_{\alpha, \omega}^{p}}^{p}\right)^{1 / p} \\
& \asymp\left(\left\|D^{(n)}\right\|_{\mathcal{F}_{\alpha, \omega}^{p} \rightarrow L^{q}(\mu)}+C_{\mu, n}\right)\|f\|_{\mathcal{F}_{\alpha, \omega n p}^{p}} .
\end{aligned}
$$

So, $I_{d}: \mathcal{F}_{\alpha, \omega_{n p}}^{p} \rightarrow L^{q}(\mu)$ is bounded and

$$
\left\|I_{d}\right\|_{\mathcal{F}_{\alpha, \omega_{n p}}^{p} \rightarrow L^{q}(\mu)} \lesssim\left\|D^{(n)}\right\|_{\mathcal{F}_{\alpha, \omega}^{p} \rightarrow L^{q}(\mu)}+C_{\mu, n} .
$$

Finally, assume that $I_{d}: \mathcal{F}_{\alpha, \omega_{n p}}^{p} \rightarrow L^{q}(\mu)$ is bounded, that is,

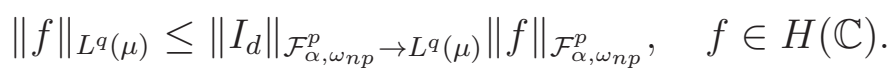

Then, replacing in the above inequality $f$ by $D^{(n)}(f)$ and applying Theorem $1.1\left(\right.$ with $\left.\omega_{n p}\right)$, it follows that

$\left\|D^{(n)}(f)\right\|_{L^{q}(\mu)} \leq\left\|I_{d}\right\|_{\mathcal{F}_{\alpha, \omega_{n p}}^{p} \rightarrow L^{q}(\mu)}\left\|D^{(n)}(f)\right\|_{\mathcal{F}_{\alpha, \omega_{n p}}^{p}} \asymp\left\|I_{d}\right\|_{\mathcal{F}_{\alpha, \omega_{n} p}^{p} \rightarrow L^{q}(\mu)}\|f\|_{\mathcal{F}_{\alpha, \omega}^{p}}$.

So, $D^{(n)}: \mathcal{F}_{\alpha, \omega}^{p} \rightarrow L^{q}(\mu)$ is bounded and $\left\|D^{(n)}\right\|_{\mathcal{F}_{\alpha, \omega}^{p} \rightarrow L^{q}(\mu)} \lesssim\left\|I_{d}\right\|_{\mathcal{F}_{\alpha, \omega_{n p}}^{p} \rightarrow L^{q}(\mu)}$. Consequently, (i) $\Leftrightarrow($ ii) $\Leftrightarrow$ (iii), as well as (1.3) and (1.5) hold for any negative integer $n$. This finishes the proof.

\section{Pointwise multipliers And Embeddings}

In this section, by using Theorem 1.2 (with $n=0$ ) we provide descriptions of the space of pointwise multipliers $\mathcal{F}_{\alpha, \omega}^{p}$ to $\mathcal{F}_{\beta, \eta}^{q}$, where $0<q, p<\infty$ and

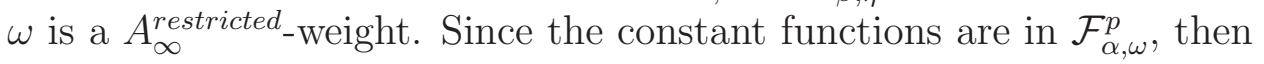

$$
\operatorname{Mult}\left(\mathcal{F}_{\alpha, \omega}^{p}, \mathcal{F}_{\beta, \eta}^{q}\right) \subset \mathcal{F}_{\beta, \eta}^{q} .
$$

In fact, it follows from the closed graph theorem that

$g \in \operatorname{Mult}\left(\mathcal{F}_{\alpha, \omega}^{p}, \mathcal{F}_{\beta, \eta}^{q}\right)$ if and only if

$$
d \mu_{g}(z):=|g(z)|^{q} e^{-q \frac{\beta}{2}|z|^{2}} \eta(z) d A(z)
$$

is a $q$-Carleson measure for $\mathcal{F}_{\alpha, \omega}^{p}$. Thus, Theorem 1.2 yields the following result.

Corollary 5.1. Let $\alpha, \beta>0, \omega \in A_{\infty}^{\text {restricted }}$ and $\eta$ be a weight. If $g \in \mathcal{F}_{\beta, \eta}^{q}$, then 
(i) If $0<p \leq q<\infty, g \in \operatorname{Mult}\left(\mathcal{F}_{\alpha, \omega}^{p} \rightarrow \mathcal{F}_{\beta, \eta}^{q}\right)$ if and only if there exists $C>0$ such that

$$
G(u):=\frac{1}{\omega(D(u, 1))} \int_{D(u, 1)}|g(z)|^{q} e^{-q \frac{\beta-\alpha}{2}|z|^{2}} \eta(z) d A(z) \leq C \omega(D(u, 1))^{(q-p) / p},
$$

for any $u \in \mathbb{C}$.

(ii) If $0<q<p<\infty, g \in \operatorname{Mult}\left(\mathcal{F}_{\alpha, \omega}^{p}, \mathcal{F}_{\beta, \eta}^{q}\right)$ if and only if $G \in$ $L^{p /(p-q)}(\mathbb{C}, \omega)$.

In particular, taking $g=1$ in the above corollary we obtain the next result.

Corollary 5.2. Let $\alpha, \beta>0, \omega \in A_{\infty}^{\text {restricted }}$ and $\eta$ be a weight. Then,

(i) If $0<p \leq q<\infty, \mathcal{F}_{\alpha, \omega}^{p} \subset \mathcal{F}_{\beta, \eta}^{q}$ if and only if there exists $C>0$ such that

$$
G_{1}(u):=\frac{1}{\omega(D(u, 1))} \int_{D(u, 1)} e^{-q \frac{\beta-\alpha}{2}|z|^{2}} \eta(z) d A(z) \leq C \omega(D(u, 1))^{(q-p) / p},
$$

for any $u \in \mathbb{C}$.

(ii) If $0<q<p<\infty, \mathcal{F}_{\alpha, \omega}^{p} \subset \mathcal{F}_{\beta, \eta}^{q}$ if and only if $G_{1} \in L^{p /(p-q)}(\mathbb{C}, \omega)$.

Despite the conditions in Corollary 5.1 are useful in praxis, let us see they are particularly neat when both weights coincide.

Proposition 5.3. If $\beta<\alpha$, then $\operatorname{Mult}\left(\mathcal{F}_{\alpha, \omega}^{p}, \mathcal{F}_{\beta, \omega}^{q}\right)=0$.

Proof. Let $\delta \in\left(0, \min \left\{\alpha, \frac{\alpha-\beta}{2}\right\}\right)$. By Proposition 3.3, $\mathcal{F}_{\alpha-\delta}^{\infty} \subset \mathcal{F}_{\alpha, \omega}^{p}$ and $\mathcal{F}_{\beta, \omega}^{q} \subset \mathcal{F}_{\beta+\delta}^{\infty}$, so

$$
\operatorname{Mult}\left(\mathcal{F}_{\alpha, \omega}^{p}, \mathcal{F}_{\beta, \omega}^{q}\right) \subset \operatorname{Mult}\left(\mathcal{F}_{\alpha-\delta}^{\infty}, \mathcal{F}_{\beta+\delta}^{\infty}\right) .
$$

For each $a \in \mathbb{C}$ consider the function $f_{a}(z)=e^{(\alpha-\delta) z \bar{a}}$. Since

$$
\left|f_{a}(z)\right| e^{-(\alpha-\delta)|z|^{2} / 2} \leq e^{(\alpha-\delta)|a|^{2} / 2}=\left\|f_{a}\right\|_{\mathcal{F}_{\alpha-\delta}^{\infty}},
$$

for any $g \in \operatorname{Mult}\left(\mathcal{F}_{\alpha-\delta}^{\infty}, \mathcal{F}_{\beta+\delta}^{\infty}\right)$,

$$
\begin{aligned}
|g(a)| e^{(2 \alpha-\beta-3 \delta)|a|^{2} / 2} & =\left|g(a) f_{a}(a)\right| e^{-(\beta+\delta)|a|^{2} / 2} \leq\left\|g f_{a}\right\|_{\mathcal{F}_{\beta+\delta}^{\infty}} \\
& \lesssim\left\|f_{a}\right\|_{\mathcal{F}_{\alpha-\delta}^{\infty}}=e^{(\alpha-\delta)|a|^{2} / 2},
\end{aligned}
$$

which gives $|g(a)| \lesssim e^{(\beta-\alpha+2 \delta)|a|^{2} / 2}$.

Since $\beta-\alpha+2 \delta<0$, the maximum modulus principle gives $g=0$.

Theorem 5.4. Let $\alpha, \beta>0$ and $\omega \in A_{\infty}^{\text {restricted }}$. Then,

(i) If $\alpha<\beta$, then $\operatorname{Mult}\left(\mathcal{F}_{\alpha, \omega}^{p}, \mathcal{F}_{\beta, \omega}^{p}\right)=\mathcal{F}_{\beta-\alpha}^{\infty}$;

(ii) $\operatorname{Mult}\left(\mathcal{F}_{\alpha, \omega}^{p}, \mathcal{F}_{\alpha, \omega}^{p}\right)=\mathbb{C}$;

(iii) If $q>p$ and $\alpha \leq \beta$, then $g \in \operatorname{Mult}\left(\mathcal{F}_{\alpha, \omega}^{p}, \mathcal{F}_{\beta, \omega}^{q}\right)$ if and only if

$$
|g(u)| e^{\frac{\alpha-\beta}{2}|u|^{2}} \lesssim \omega(D(u, 1))^{(q-p) / p q} ;
$$

(iv) If $p>q>0$ and $\alpha<\beta$, then $\operatorname{Mult}\left(\mathcal{F}_{\alpha, \omega}^{p}, \mathcal{F}_{\beta, \omega}^{q}\right)=\mathcal{F}_{\beta-\alpha, \omega}^{p q /(p-q)}$; 
(v) If $p>q>0$, then $\operatorname{Mult}\left(\mathcal{F}_{\alpha, \omega}^{p}, \mathcal{F}_{\alpha, \omega}^{q}\right)=H(\mathbb{C}) \cap L^{p q /(p-q)}(\mathbb{C}, \omega)$.

Proof. Bearing in mind Corollary 5.1, in each case (i)-(v), it is easy to see that the condition describing $\operatorname{Mult}\left(\mathcal{F}_{\alpha, \omega}^{p}, \mathcal{F}_{\alpha, \omega}^{q}\right)$ is sufficient. Indeed, in order to prove this implication in (i), (ii) and (iii), it is enough to use these conditions to prove that the corresponding function $G$ satisfies the inequality in Corollary 5.1(i). In (iii), we also use that $\omega(D(z, 1)) \asymp \omega(D(u, 1))$ for any $z \in D(u, 1)$ (see Lemma B). The analogue implications in (iv), (v) follow from Corollary 5.1(ii) and Hölder's inequality with exponent $p / q$.

In each case (i)-(v), the reverse direction follows from Corollary 5.1 and Lemma 3.1, which provides the pointwise estimate

$$
|g(u)| e^{\frac{\alpha-\beta}{2}|u|^{2}} \lesssim G(u)^{1 / q}, \quad \beta \geq \alpha .
$$

In particular, this estimate in (ii) gives $\operatorname{Mult}\left(\mathcal{F}_{\alpha, \omega}^{p}, \mathcal{F}_{\alpha, \omega}^{p}\right) \subset H(\mathbb{C}) \cap L^{\infty}$, which, by Liouville's theorem, coincides with $\mathbb{C}$.

It is worth mentioning that Proposition 5.3 and Theorem 5.4, together with Theorem 1.1, provide descriptions of pointwise multipliers between

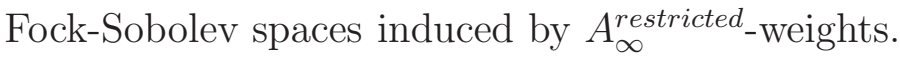

\section{REFERENCES}

[1] A. Aleman and O. Constantin, Spectra of integration operators on weighted Bergman spaces, J. Anal. Math. 109 (2009), 199-231.

[2] D. Bekollé and A. Bonami, Inégalités á poids pour le noyau de Bergman, (French) C. R. Acad. Sci. Paris Sr. A-B 286 (1978), no. 18, 775-778.

[3] H. R. Cho and K. H. Zhu, Fock Sobolev spaces and their Carleson measures, J. Funct. Anal. 263 (2012), 2483-2506.

[4] O. Constantin, A Volterra-type integration operator on Fock spaces, Proc. Amer. Math. Soc. 140 (2012), 4247-4257.

[5] O. Constantin and J. A.Peláez, Integral operators, Embedding theorems and a Littlewood-Paley formula on Fock spaces, J. Geom. Anal 26 (2016), no. 2, 11091154.

[6] J. Duoandikoetxea, Fourier Analysis, Graduate Studies in Mathemticds, 29, Amer. Math. Soc., Providence, RI 2001.

[7] J. Duoandikoetxea, F. J Martin-Reyes and S. Ombrosi, On the $A_{\infty}$ conditions for general bases, Math. Z., 282 (2016), no. 3, 955-972.

[8] P.L. Duren, Theory of $H^{p}$ Spaces, Academic Press, New York-London (1970). Reprint: Dover, Mineola, New York (2000).

[9] J. Garcia-Cuerva and J.L. Rubio de Francia, Weighted Norm Inequalities and related Topics, North-Holland Mathematics Studies, 116. North-Holland Publishing Co., Amsterdam, (1985).

[10] J. Isralowitz, Invertible Toeplitz products, weighted norm inequalities, and $A_{p}$ weights, J. Oper. Theory 71, (2014), no. 2, 381-410.

[11] J. Isralowitz and K. Zhu, Toeplitz operators on the Fock space, Int. Equ. Oper. Th. 66 (2010), no. 4, 593-611.

[12] R. A. Kerman and A. Torchinsky, Integral inequalities with weights for the maximal function, Studia Math. 71 (1981/82), no. 3, 277-284.

[13] D. H. Luecking, Embedding theorems for spaces of analytic functions via Khinchine's inequality, Michigan Math. J. 40 (1993), 333-358. 
[14] N. Marco, M. Massaneda and J. Ortega-Cerdà, Interpolating and sampling sequences for entire functions, Geom. Funct. Anal. 13 (2003), 862-914.

[15] T. Mengestie, Carleson measures for Fock-Sobolev spaces, Complex Anal. Oper. Theory 8 (2014), no. 6, 1225-1256.

[16] J. Ortega-Cerdá and K. Seip, Beurling-type density theorems for weighted $L^{p}$ spaces of entire functions, J. Anal. Math. 75 (1998), 247-266.

[17] J. A. Peláez and J. Rättyä, Two weight inequality for Bergman projection, J. Math. Pures Appl. (9) 105 (2016), no 1, 102-130.

[18] E. M. Stein, Harmonic Analysis: Real-Variable Methods, Orthogonality, and Oscillatory integrals, Princeton Univ. Press (1993).

[19] J. Tung, Fock Spaces, PhD dissertation, University of Michigan, 2005.

[20] K. Zhu, Operator Theory in Function Spaces, Second Edition, Math. Surveys and Monographs, 138, American Mathematical Society: Providence, Rhode Island, (2007).

[21] K. Zhu, Analysis on Fock Spaces, Springer-Verlag, New York, (2012).

Carme Cascante, Departament de Matemàtica Aplicada i Analisi, Universitat de Barcelona, Gran Via 585, 08007 Barcelona, Spain

E-mail address: cascante@ub.edu

Joan Fàbrega, Departament de Matemàtica Aplicada i Analisi, Universitat de Barcelona, Gran Via 585, 08007 Barcelona, Spain

E-mail address: joan_fabrega@ub.edu

José A. Peláez, Departamento de Análisis Matemático, Facultad de CienCias, 29071, Málaga, Spain.

E-mail address: japelaez@uma.es 